\title{
A New Class of Generalized Modified Weibull Distribution with Applications
}

\author{
Broderick O. Oluyede \\ Shujiao Huang \\ Tiantian Yang \\ Georgia Southern University Georgia Southern University Georgia Southern University
}

\begin{abstract}
A new five parameter gamma-generalized modified Weibull (GGMW) distribution which includes exponential, Rayleigh, Weibull, modified Weibull, gamma-modified Weibull, gamma-modified Rayleigh, gamma-modified exponential, gamma-Weibull, gamma-Rayleigh, gamma-linear failure rate and gamma-exponential distributions as special cases is proposed and studied. Some mathematical properties of the new class of distributions including hazard function, quantile function, moments, distribution of the order statistics and Rényi entropy are presented. Maximum likelihood estimation technique is used to estimate the model parameters and applications to real datasets in order to illustrate the usefulness of the proposed class of models are presented.
\end{abstract}

Keywords: Gamma distribution, Modified Weibull distribution, Maximum likelihood estimation.

\section{Introduction}

Weibull distribution has been widely used for modeling data in a wide variety of areas including reliability, engineering, stochastic processes, survival analysis and renewal theory. In this paper, we present and study the mathematical properties of the gamma-generalized modified Weibull distribution. This class of distributions is flexible in accommodating all forms of hazard rate functions and contains several well known and new sub-models such as Weibull, Rayleigh, exponential, modified Weibull, gamma-modified Weibull, gamma-modified exponential, gamma-Weibull, gamma-Rayleigh, gamma-linear failure rate, gamma-extreme value, gamma-additive exponential and gamma-exponential distributions.

There are several extensions of the Weibull distribution and its sub-models including the exponentiated Weibull (Mudholkar, Srivastava, and Kollia 1996), which is a special case of the beta Weibull distribution proposed by (Lee, Famoye, and Olumolade 2007), generalized Rayleigh (Kundu and Rakab 2005), exponentiated exponential (Gupta and Kundu 1999), (Gupta and Kundu 2001), modified Weibull (Mudholkar, Srivastava, and Friemer 1995), exponentiated modified Weibull (Sarhan and Zaindin 2009), and a host of other distributions, some of which are presented in section 2 of this paper. Additional generalizations of Weibull distribution include (Famoye, Lee, and Olumolade 2005) where the authors discussed and presented results on the beta-Weibull distribution. (Nadarajah 2005) presented results on the modified Weibull 
distribution. A host of researchers have also developed several parameter Weibull, modified Weibull and flexible Weibull distributions over the years. The two parameter Weibull extensions include (Bebbington, Lai, and Zitikis 2007), (Zhang and Xie 2011). The three parameter Weibull extensions include (Marshall and Olkin 1997), (Xie, Tang, and Goh 2002), (Nadarajah and Kotz 2005). Some of these extensions enable the accommodation of bathtub shape hazard rate function. (Carrasco, Ortega, and Cordeiro 2008) generalized the modified Weibull distribution of (Lai, Moore, and Xie 1998) to obtain the exponentiated modified Weibull distribution. The four parameter generalizations include the additive Weibull distribution of (Xie and Lai 1995), modified Weibull (Sarhan and Zaindin 2009), beta-Weibull proposed by (Famoye et al. 2005) and Kumaraswamy Weibull by (Cordeiro, Ortega, and Nadarajah 2010). The five parameter modified Weibull distribution include those introduced by (Phani 1987), beta modified Weibull by (Silva, Ortega, and Cordeiro 2010) and (Nadarajah, Cordeiro, and Ortega 2011). Additional results on the generalization of the Weibull distribution include work by (Singha, Jain, and Kumar 2012), as well as (Almalki and Yuan 2013) where results on a new modified Weibull distribution was presented. (Barlow and Campo 1975) discussed total time on test processes with application to failure data analysis. (Choudhury 2005) presented moments of the exponentiated Weibull distribution. The exponentiated Weibull distribution was also studied by (Nassar and Eissa 2003). (Haupt and Schabe 1992) presented a model for bathtub shaped failure rate function. (Hjorth 1980) studied a reliability function with increasing, decreasing and bathtub shaped failure rate functions, and (Rajarshi and Rajarshi 1988) gave a comprehensive review of bathtub shaped distributions.

For any continuous baseline cdf $F(x)$, and $x \in \mathbf{R}$, (Zografos and Balakrishnan 2009) defined the distribution (when $\psi=1$ in equation (1)) with pdf $g(x)$ and cdf $G(x)$ (for $\delta>0$ ) as follows:

$$
g(x)=\frac{1}{\Gamma(\delta) \psi^{\delta}}[-\log (\bar{F}(x))]^{\delta-1}(1-F(x))^{1 / \psi-1} f(x),
$$

and

$$
G(x)=\frac{1}{\Gamma(\delta) \psi^{\delta}} \int_{0}^{-\log (\bar{F}(x))} t^{\delta-1} e^{-t / \psi} d t=\frac{\gamma\left(\delta,-\psi^{-1} \log (\bar{F}(x))\right)}{\Gamma(\delta)},
$$

respectively, where $g(x)=d G(x) / d x, \Gamma(\delta)=\int_{0}^{\infty} t^{\delta-1} e^{-t} d t$ is the gamma function, and $\gamma(z, \delta)=\int_{0}^{z} t^{\delta-1} e^{-t} d t$ is the incomplete gamma function. The corresponding hazard rate function (hrf) is

$$
h_{G}(x)=\frac{[-\log (1-F(x))]^{\delta-1} f(x)(1-F(x))^{1 / \psi-1}}{\psi^{\delta}\left(\Gamma(\delta)-\gamma\left(-\psi^{-1} \log (1-F(x)), \delta\right)\right)} .
$$

When $\psi=1$, this distribution is referred to as the ZB-G family of distributions. Also, (when $\psi=1$ ), (Ristić and Balakrishnan 2011) proposed an alternative gamma-generator defined by the cdf and pdf

$$
G_{2}(x)=1-\frac{1}{\Gamma(\delta) \psi^{\delta}} \int_{0}^{-\log F(x)} t^{\delta-1} e^{-t / \psi} d t, \quad x \in \mathbf{R}, \delta>0
$$

and

$$
g_{2}(x)=\frac{1}{\Gamma(\delta) \psi^{\delta}}[-\log (F(x))]^{\delta-1}(F(x))^{1 / \psi-1} f(x),
$$

respectively. Note that if $\psi=1$ and $\delta=n+1$, in equations (1) and (2), we obtain the cdf and pdf of the upper record values $U$ given by

$$
G_{U}(u)=\frac{1}{n !} \int_{0}^{-\log (1-F(u))} y^{n} e^{-y} d y
$$

and

$$
g_{U}(u)=f(u)[-\log (1-F(u))]^{n} / n !
$$


Similarly, from equations (4) and (5), the pdf of the lower record values $T$ is given by

$$
g_{L}(t)=f(t)[-\log (F(t))]^{n} / n ! .
$$

In this paper, we will consider and present a generalization of the generalized modified Weibull distribution via the family of distributions given in equation (5). (Zografos and Balakrishnan 2009) motivated the ZB-G model as follows. Let $X_{(1)}, X_{(2)}, \ldots \ldots, X_{(n)}$ be upper record values from a sequence of independent and identically distributed (i.i.d.) random variables from a population with pdf $f(x)$. Then, the pdf of the $n^{t h}$ upper record value is given by equation (1) when $\psi=1$. A logarithmic transformation of the parent distribution $F$ transforms the random variable $X$ with density (1) to a gamma distribution. That is, if $\mathrm{X}$ has the density (1), then the random variable $Y=-\log [1-F(X)]$ has a gamma distribution $G A M(\delta ; 1)$ with density $k(y ; \delta)=\frac{1}{\Gamma(\delta)} y^{\delta-1} e^{-y}, y>0$. The opposite is also true, if $Y$ has a gamma $\operatorname{GAM}(\delta ; 1)$ distribution, then the random variable $X=G^{-1}\left(1-e^{-Y}\right)$ has a ZB-G distribution. In addition to the motivations provided by (Zografos and Balakrishnan 2009), we are interested in the generalization of the generalized modified Weibull distribution via the gamma-generator and establishing the relationship between weighted distributions and equations (1) and (5), respectively.

Weighted distributions applies to a variety of areas and provides an approach to dealing with model specification and data interpretation problems. It adjusts the probabilities of actual occurrence of events to arrive at a specification of the probabilities when those events are recorded. (Fisher 1934) introduced the concept of weighted distribution, in order to study the effect of ascertainment upon estimation of frequencies. (Patil and Rao 1978) used weighted distribution as stochastic models in the study of harvesting and predation. (Rao 1965) unified concept of weighted distribution and use it to identify various sampling situations. The usefulness and applications of weighted distribution to biased samples in various areas including medicine, ecology, reliability, and branching processes can also be seen in (Nanda and Jain 1999), (Gupta and Keating 1985), (Oluyede 1999) and in references therein. Let $Y$ be a non-negative random variable with its natural $\operatorname{pdf} f(y ; \underline{\theta})$, where $\underline{\theta}$ is a vector of parameters, then the pdf of the weighted random variable $Y^{w}$ is given by:

$$
f^{w}(y ; \underline{\theta}, \underline{\beta})=\frac{w(y, \underline{\beta}) f(y ; \underline{\theta})}{\omega},
$$

where the weight function $w(y, \beta)$ is a non-negative function, that may depend on the vector of parameters $\underline{\beta}$, and $0<\omega=E(w(Y, \underline{\beta}))<\infty$ is a normalizing constant. In general, consider the weight function $w(y)$ defined as follows:

$$
w(y)=y^{k} e^{l y} F^{i}(y) \bar{F}^{j}(y) .
$$

Setting $k=0 ; k=j=i=0 ; l=i=j=0 ; k=l=0 ; i \rightarrow i-1 ; j=n-i ; k=l=i=0$ and $k=l=j=0$ in this weight function, one at a time, implies probability weighted moments, moment-generating functions, moments, order statistics, proportional hazards and proportional reversed hazards, respectively, where $F(y)=P(Y \leq y)$ and $\bar{F}(y)=1-F(y)$. If $w(y)=y$, then $Y^{*}=Y^{w}$ is called the size-biased version of $Y$.

(Ristić and Balakrishnan 2011) provided motivations for the family of distributions given in equation (4) when $\psi=1$, that is for $n \in \mathbf{N}$, equation (4) is the pdf of the $n^{\text {th }}$ lower record value of a sequence of i.i.d. variables from a population with density $f(x)$. (Ristić and Balakrishnan 2011) used the exponentiated exponential (EE) distribution with cdf $F(x)=\left(1-e^{-\beta x}\right)^{\alpha}$, where $\alpha>0$ and $\beta>0$, to obtained and study the gamma-exponentiated exponential (GEE) model. See references therein for additional results on the GEE model. In this note, we obtain a natural extension of the generalized modified Weibull distribution, which we refer to as gamma-generalized modified Weibull (GGMW) distribution.

In section 2, some basic results, the gamma-generalized modified Weibull (GGMW) distribution, series expansion and its sub-models, quantile function, hazard and reverse hazard 
functions are presented. Moments and moment generating function are given in section 3 . Section 4 contains some additional useful results on the distribution of order statistics and Rényi entropy. In section 5, results on the estimation of the parameters of the GGMW distribution via the method of maximum likelihood are presented. Applications are given in section 6 , and concluding remarks in section 7 .

\section{GGMW distribution, series expansion and sub-models}

In this section, the GGMW distribution and some of its sub-models are presented. First consider the generalized modified Weibull (GMW) distribution (Sarhan and Zaindin 2009) given by

$$
F_{G M W}(x, \alpha, \beta, \theta, \lambda)=1-\exp \left(-\alpha x-\beta x^{\theta} e^{\lambda x}\right), \quad x \geq 0, \alpha, \beta, \theta, \lambda \geq 0 .
$$

We note that in (Sarhan and Zaindin 2009) paper, the parameter $\lambda$ was taken to be zero. The parameters $\alpha$ and $\beta$ control the scale of the distribution, $\theta$ controls the shape, whereas $\lambda$ can be considered to be an accelerating factor in the imperfection time and a factor of fragility in the survival of the individual as time increases. By inserting the GMW distribution in equation (4), the survival function $\bar{G}_{G G M W}(x)=1-G_{G G M W}(x)$ of the GGMW distribution is obtained as follows:

$$
\begin{aligned}
\bar{G}_{G G M W}(x) & =\frac{1}{\Gamma(\delta) \psi^{\delta}} \int_{0}^{-\log \left(1-e^{-\alpha x-\beta x^{\theta} e^{\lambda x}}\right)} t^{\delta-1} e^{-t / \psi} d t \\
& =\frac{\gamma\left(-\psi^{-1} \log \left(1-e^{-\alpha x-\beta x^{\theta} e^{\lambda x}}\right), \delta\right)}{\Gamma(\delta)}
\end{aligned}
$$

where $x>0, \alpha, \beta, \theta, \lambda \geq 0, \delta>0, \psi>0$, and $\gamma(x, \delta)=\int_{0}^{x} t^{\delta-1} e^{-t} d t$ is the lower incomplete gamma function. The corresponding pdf is given by

$$
\begin{aligned}
g_{G G M W}(x) & =\frac{1}{\Gamma(\delta) \psi^{\delta}}\left[-\log \left(1-e^{-\alpha x-\beta x^{\theta} e^{\lambda x}}\right)\right]^{\delta-1} \\
& \times\left(\alpha+\beta x^{\theta-1} e^{\lambda x}[\theta+\lambda x]\right) e^{-\alpha x-\beta x^{\theta} e^{\lambda x}} \\
& \times\left[1-e^{-\alpha x-\beta x^{\theta} e^{\lambda x}}\right]^{(1 / \psi)-1} .
\end{aligned}
$$

If $F(x)=\left[1-e^{-\alpha x^{\eta}-\beta x^{\theta} e^{\lambda x}}\right]^{\phi}$, then the corresponding generalized gamma-generalized modified Weibull pdf is given by

$$
\begin{aligned}
g_{G G M W}(x) & =\frac{\phi}{\Gamma(\delta) \psi^{\delta}}\left[-\log \left(1-e^{-\alpha x^{\eta}-\beta x^{\theta} e^{\lambda x}}\right)^{\phi}\right]^{\delta-1} \\
& \times\left(\alpha \eta x^{\eta-1}+\beta x^{\theta-1} e^{\lambda x}[\theta+\lambda x]\right) e^{-\alpha x^{\eta}-\beta x^{\theta} e^{\lambda x}} \\
& \times\left[1-e^{-\alpha x^{\eta}-\beta x^{\theta} e^{\lambda x}}\right]^{\phi+(1 / \psi)-2} .
\end{aligned}
$$

In this note, we take $\phi=\eta=\psi=1$. The pdf in equation (14) is now given by

$$
\begin{aligned}
g_{G G M W}(x) & =\frac{1}{\Gamma(\delta)}\left[-\log \left(1-e^{-\alpha x-\beta x^{\theta} e^{\lambda x}}\right)\right]^{\delta-1} \\
& \times\left(\alpha+\beta x^{\theta-1} e^{\lambda x}[\theta+\lambda x]\right) e^{-\alpha x-\beta x^{\theta} e^{\lambda x}} .
\end{aligned}
$$

If a random variable $X$ has the GGMW density given in equation (15), we write $X \sim$ $G G M W(\alpha, \beta, \theta, \lambda, \delta)$. The parameter $\delta$ is an extra shape parameter in the GGMW distribution. Let $y=e^{-\alpha x-\beta x^{\theta} e^{\lambda x}}, 0<y<1, \alpha, \beta, \theta, \delta>0$, and $\lambda \geq 0$, then using the series representation $-\log (1-y)=\sum_{i=0}^{\infty} \frac{y^{i+1}}{i+1}$, we have

$$
[-\log (1-y)]^{\delta-1}=y^{\delta-1}\left[\sum_{m=0}^{\infty}\left(\begin{array}{c}
\delta-1 \\
m
\end{array}\right) y^{m}\left(\sum_{s=0}^{\infty} \frac{y^{s}}{s+2}\right)^{m}\right] .
$$


Applying the result on power series raised to a positive integer, with $a_{s}=(s+2)^{-1}$, that is,

$$
\left(\sum_{s=0}^{\infty} a_{s} y^{s}\right)^{m}=\sum_{s=0}^{\infty} b_{s, m} y^{s}
$$

where $b_{s, m}=\left(s a_{0}\right)^{-1} \sum_{l=1}^{s}[m(l+1)-s] a_{l} b_{s-l, m}$, and $b_{0, m}=a_{0}^{m}$, (Gradshteyn and Ryzhik 2000), the GGMW pdf can be written as

$$
\begin{aligned}
g_{G G M W}(x) & =\frac{1}{\Gamma(\delta)} \sum_{m=0}^{\infty} \sum_{s=0}^{\infty}\left(\begin{array}{c}
\delta-1 \\
m
\end{array}\right) b_{s, m} y^{m+s+\delta}\left(\alpha+\beta x^{\theta-1} e^{\lambda x}[\theta+\lambda x]\right) \\
& =\frac{1}{\Gamma(\delta)} \sum_{m=0}^{\infty} \sum_{s=0}^{\infty}\left(\begin{array}{c}
\delta-1 \\
m
\end{array}\right) b_{s, m} e^{-\alpha(m+s+\delta) x-\beta(m+s+\delta) x^{\theta} e^{\lambda x}} \\
& \times \frac{m+s+\delta}{m+s+\delta}\left(\alpha+\beta x^{\theta-1} e^{\lambda x}[\theta+\lambda x]\right) \\
& =\sum_{m=0}^{\infty} \sum_{s=0}^{\infty}\left(\begin{array}{c}
\delta-1 \\
m
\end{array}\right) \frac{b_{s, m}}{\Gamma(\delta)(m+s+\delta)} g_{*}(x ; \alpha(m+s+\delta), \beta(m+s+\delta), \theta, \lambda),
\end{aligned}
$$

where $g_{*}(x ; \alpha(m+s+\delta), \beta(m+s+\delta), \theta, \lambda)$ is the generalized modified Weibull pdf with parameters $\alpha(m+s+\delta)>0, \beta(m+s+\delta)>0, \theta>0$, and $\lambda \geq 0$. Let $C=\left\{(m, s) \in \mathbf{Z}_{+}^{2}\right\}$, then the weights in the GGMW pdf above are

$$
w_{\nu}=\left(\begin{array}{c}
\delta-1 \\
m
\end{array}\right) \frac{b_{s, m}}{(m+s+\delta) \Gamma(\delta)},
$$

and

$$
g_{G G M W}(x)=\sum_{\nu \in C} w_{\nu} g_{*}(x ; \alpha(m+s+\delta), \beta(m+s+\delta), \theta, \lambda),
$$

for $x>0, \delta>0, \alpha(m+s+\delta), \beta(m+s+\delta), \theta>0$, and $\lambda \geq 0$. It follows therefore that the GGMW density is linear combination of the generalized modified Weibull (GMW) densities. The statistical and mathematical properties of the GGMW distribution can be readily obtained from those of the generalized modified Weibull distribution.

For the convergence of equations (16) and (17), as well as elsewhere in this paper, note that for $\delta>0$,

$$
[-\log (1-y)]^{\delta-1}=\left[y\left(1+y \sum_{s=0}^{\infty} \frac{y^{s}}{s+2}\right)\right]^{\delta-1}
$$

so that

$$
\left[1+y \sum_{k=0}^{\infty} \frac{y^{k}}{k+2}\right]^{\delta-1}=\sum_{k=0}^{\infty}\left(\begin{array}{c}
\delta-1 \\
k
\end{array}\right) y^{k}\left(\sum_{s=0}^{\infty} \frac{y^{s}}{s+2}\right)^{k}
$$

is convergent if and only if $0<\left(y \sum_{k=0}^{\infty} \frac{y^{k}}{k+2}\right)^{k}<1 \forall y \in(0,1)$, since $0<y=e^{-\alpha x-\beta x^{\theta} e^{\lambda x}}<$ 1 , for $x>0, \alpha, \beta, \theta>0$, and $\lambda \geq 0$. Now, $y \sum_{k=0}^{\infty} \frac{y^{k}}{k+2}=\frac{-\log (1-y)}{y}-1$, so we must have $0<\frac{-\log (1-y)}{y}-1<1$. This leads to $1-y>\exp (-2 y)$, and on the other hand $\exp (-y)=$ $\sum_{k=0}^{\infty} \frac{(-1)^{k} y^{k}}{k !}>1-y$. Thus, we have the system of inequalities $1-y>\exp (-2 y)$ and $\exp (-y)>1-y$, which is satisfied $\forall y \in(0,0.7968)$. The implication here is that the inequality $0<\left(y \sum_{k=0}^{\infty} \frac{y^{k}}{k+2}\right)^{k}<1$ is not valid for all values of $0<y=e^{-\alpha x-\beta x^{\theta} e^{\lambda x}}<1$, and equations (16) and (17), and elsewhere in this paper are convergent only $\forall y \in(0,0.7968)$. The series in equations (16) and (17), and elsewhere in this paper are not valid for all values of $0<$ 

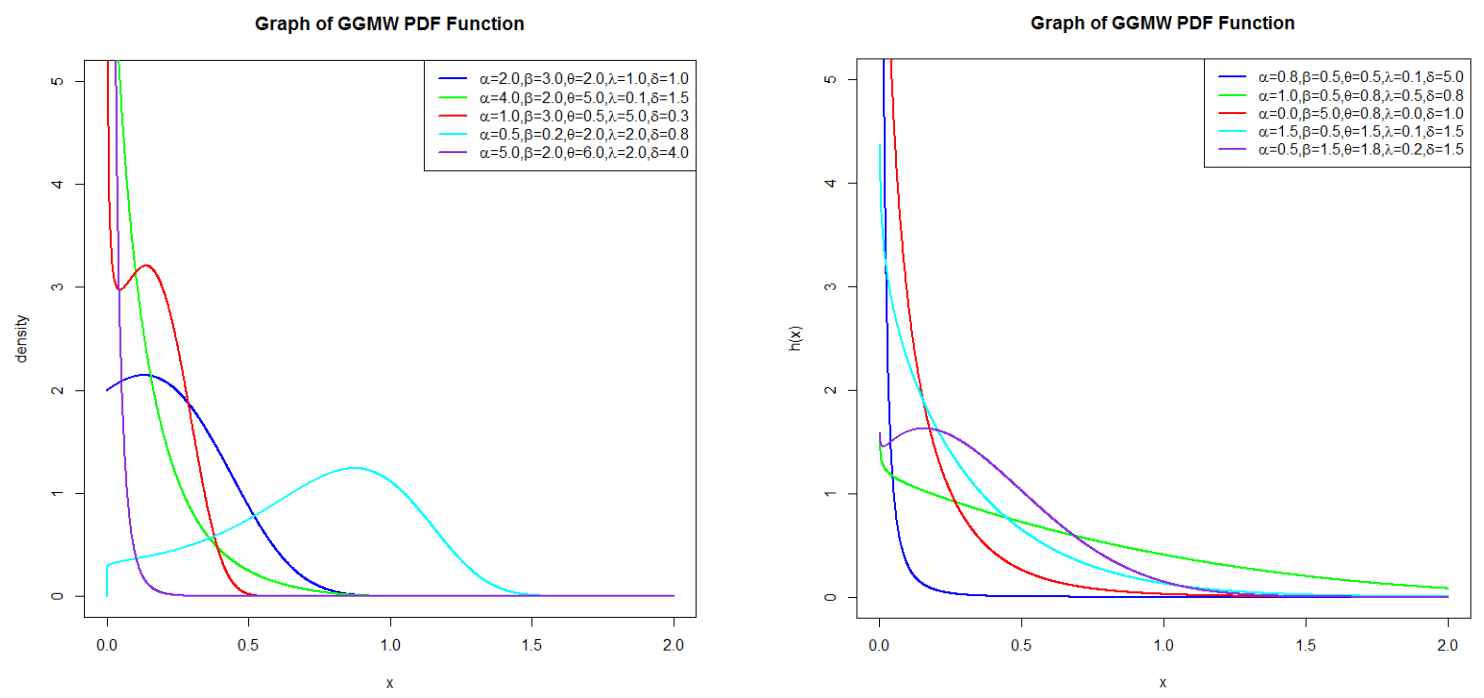

Figure 1: Graphs of GGMW pdf

$y=e^{-\alpha x-\beta x^{\theta} e^{\lambda x}}<1$, but are convergent $\forall y \in(0,0.7968)$, and not valid (convergent) for $y>0.7986$.

Note that in general, $g_{G G M W}(x)$ is a weighted pdf with the weight function

$$
w(x)=[-\log (1-F(x))]^{\delta-1}[1-F(x)]^{\frac{1}{\psi}-1},
$$

that is,

$$
\begin{aligned}
g_{G G M W}(x) & =\frac{[-\log (1-F(x))]^{\delta-1}[1-F(x)]^{\frac{1}{\psi}-1}}{\psi^{\delta} \Gamma(\delta)} f(x) \\
& =\frac{w(x) f(x)}{E_{F}(w(X))}
\end{aligned}
$$

where $0<E_{F}\left\{[-\log (1-F(x))]^{\delta-1}[1-F(x)]^{\frac{1}{\psi}-1}\right\}=\psi^{\delta} \Gamma(\delta)<\infty$, is the normalizing constant. Graphs of the pdf of GGMW distribution are given in the Figure 1 for selected values of the parameters. The plots show that the GGMW pdf can be decreasing or right skewed among several other possible shapes as seen in Figure 1. The distribution has positive asymmetry.

\subsection{Quantile function}

The quantile function of the GGMW distribution is given by the solution of the nonlinear equation

$$
\frac{\gamma\left(-\log \left[1-e^{-\alpha x-\beta x^{\theta} e^{\lambda x}}\right], \delta\right)}{\Gamma(\delta)}=1-u
$$

That is, $-\log \left[1-e^{-\alpha x-\beta x^{\theta} e^{\lambda x}}\right]=\gamma^{-1}((1-u) \Gamma(\delta), \delta)$ and

$$
\alpha x+\beta x^{\theta} e^{\lambda x}=-\log \left(1-\exp \left(-\gamma^{-1}((1-u) \Gamma(\delta), \delta)\right)\right) .
$$

We can simulate from the GGMW by solving the nonlinear equation

$$
\alpha x+\beta x^{\theta} e^{\lambda x}+\log \left(1-\exp \left(-\gamma^{-1}((1-u) \Gamma(\delta), \delta)\right)\right)=0,
$$

where $u$ is a uniformly distributed random variable on the interval $[0,1]$. The inverse incomplete gamma function can be implemented by using numerical methods. Consequently, random numbers can be generated based the equation above. Table 1 lists the quantile for selected parameter values of the GGMW distribution. 
Table 1: GGMW quantile for selected values

\begin{tabular}{cccccc}
\hline & \multicolumn{5}{c}{$(\alpha, \beta, \theta, \lambda, \delta)$} \\
\cline { 2 - 6 } $\mathrm{u}$ & $(1,1,1,1,1)$ & $(2,1,2,1,1)$ & $(6,4,3,6,1)$ & $(5,3,3,5,6)$ & $(0.1,0.3,0.4,0.2,0.3)$ \\
\hline 0.1 & 0.05132855 & 0.0512954 & 0.01755608 & 0.00001875 & 1.20674200 \\
0.2 & 0.1056817 & 0.1053998 & 0.03714788 & 0.00007372 & 2.59472200 \\
0.3 & 0.1637671 & 0.1627524 & 0.05924798 & 0.00018145 & 3.82692100 \\
0.4 & 0.226598 & 0.2240198 & 0.08447059 & 0.00037058 & 4.95229000 \\
0.5 & 0.2957024 & 0.2902609 & 0.11359275 & 0.00069065 & 6.01905300 \\
0.6 & 0.3735554 & 0.3632644 & 0.14752769 & 0.00123419 & 7.07161000 \\
0.7 & 0.4646056 & 0.4463389 & 0.18721167 & 0.00219605 & 8.16167800 \\
0.8 & 0.5783069 & 0.5466338 & 0.23367423 & 0.00407477 & 9.37370000 \\
0.9 & 0.7424909 & 0.6853097 & 0.29044828 & 0.00874208 & 10.92629700 \\
\hline
\end{tabular}

\subsection{Some sub-models of the GGMW distribution}

The proposed model has several new and well known sub-models. Some of the sub-models of the GGMW distribution are listed in Table 2. They include the gamma-generalized modified Rayleigh (GGMR), gamma-generalized modified exponential (GGME), gamma-modified Weibull (GMW), gamma-modified exponential (GME), gamma-additive exponential (GAE), gamma-extreme value (GEV), gamma-Weibull (GW), modified Weibull (MW), Sardin and Zaindin modified Weibull (S-ZMW), modified Rayleigh (MR), modified exponential (ME), gamma-linear failure rate (GLFR), linear failure rate (LFR), extreme value (EV), Weibull (W) and exponential (E) distributions.

\subsection{Hazard and reverse hazard functions}

In this section, we present the hazard and reverse hazard functions, as well as graphs of the hazard function for selected values of the model parameters. Let $X$ be a continuous random variable with distribution function $G$, and probability density function (pdf) $g$, then the hazard function, reverse hazard function and mean residual life functions are given by $h_{G}(x)=g(x) / \bar{G}(x), \tau_{G}(x)=g(x) / G(x)$, and $\delta_{G}(x)=\int_{x}^{\infty} \bar{G}(u) d u / \bar{G}(x)$, respectively. The functions $\lambda_{G}(x), \delta_{G}(x)$, and $\bar{G}(x)$ are equivalent. (Shaked and Shanthikumar 1994). The hazard and reverse hazard functions are of the GGMW distribution are given by

$$
h_{G}(x)=\frac{\left\{-\log \left(1-e^{-\alpha x-\beta x^{\theta} e^{\lambda x}}\right)\right\}^{\delta-1} e^{-\alpha x-\beta x^{\theta} e^{\lambda x}}\left(\alpha+\beta x^{\theta-1} e^{\lambda x}[\theta+\lambda x]\right)}{\gamma\left(-\log \left(1-e^{-\alpha x-\beta x^{\theta} e^{\lambda x}}\right), \delta\right)},
$$

and

$$
\tau_{G}(x)=\frac{\left\{-\log \left(1-e^{-\alpha x-\beta x^{\theta} e^{\lambda x}}\right)\right\}^{\delta-1} e^{-\alpha x-\beta x^{\theta} e^{\lambda x}}\left(\alpha+\beta x^{\theta-1} e^{\lambda x}[\theta+\lambda x]\right)}{\Gamma(\delta)-\gamma\left(-\log \left(1-e^{-\alpha x-\beta x^{\theta} e^{\lambda x}}\right), \delta\right)},
$$

respectively. Plots of the hazard rate function for different combinations of the parameter values are given in Figure 2. The plot shows various shapes including monotonically increasing, monotonically increasing and bathtub shapes for five combinations of the values of the parameters. This flexibility makes the GGMW hazard rate function suitable for both monotonic and non-monotonic empirical hazard behaviors that are likely to be encountered in real life situations.

\section{Moments and moment generating function}

In this section, we obtain moments and moment generating function of the GGMW distribution. Let $X \sim G G M W(\alpha, \beta, \theta, \lambda, \delta)$, and $Y \sim G M W(\alpha, \beta, \theta, \lambda)$. Note that the $r^{t h}$ moment of the random variable $Y$ is obtained as follows. By Taylor series expansion of the functions 
Table 2: Sub-models of the gamma generalized modified Weibull distribution

\begin{tabular}{|c|c|c|c|c|c|c|c|}
\hline Model & $\alpha$ & & $\theta$ & $\lambda$ & & $G(x)$ & Reference \\
\hline GGMR & 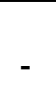 & & 2 & & & $\frac{\gamma\left(-\log \left[1-e^{-\alpha x-\beta x^{2}} e^{x x}\right], \delta\right)}{\Gamma(\delta)}$ & New \\
\hline GGNE & - & - & 1 & - & & $\frac{\gamma\left(-\log \left[1-e^{-\alpha x-\beta x e^{\lambda x}}\right], \delta\right)}{\Gamma(\delta)}$ & New \\
\hline GMW & 0 & - & - & - & & $\frac{\gamma\left(-\log \left[1-e^{-\beta x x^{\theta} e^{\lambda x}}\right], \delta\right)}{\Gamma(\delta)}$ & New \\
\hline GME & 0 & - & 1 & - & . & $\frac{\gamma\left(-\log \left[1-e^{-\beta x e^{\lambda x}}\right], \delta\right)}{\Gamma(\delta)}$ & New \\
\hline GAE & - & - & 1 & & & $\frac{\gamma\left(-\log \left[1-e^{\alpha x-\beta x}\right], \delta\right)}{\Gamma(\delta)}$ & New \\
\hline GEV & 0 & 1 & 0 & & & $\frac{\gamma\left(-\log \left[1-e^{-e^{\lambda x}}\right], \delta\right)}{\Gamma(\delta)}$ & New \\
\hline GW & 0 & - & - & 0 & 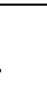 & $\frac{\gamma\left(-\log \left[1-e^{-\beta x^{\theta}}\right], \delta\right)}{\Gamma(\delta)}$ & Pinho, Cordeiro, and Nobre (2012) \\
\hline MW & 0 & - & - & - & . & $1-e^{-\beta x^{\theta} e^{\lambda x}}$ & Lai, Xie, and Murthy (2003) \\
\hline S-ZMW & - & - & - & - & . & $1-e^{-\alpha x-\beta x^{\theta} e^{\lambda x}}$ & Sarhan and Zaindin (2009) \\
\hline LFR & - & - & 2 & 0 & . & $1-e^{-\alpha x-\beta x^{2}}$ & Bain (1974) \\
\hline EV & 0 & 1 & 0 & - & . & $1-e^{-e^{\lambda x}}$ & Bain (1974) \\
\hline Weibull & 0 & - & - & 0 & 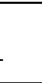 & $1-e^{-\beta x^{\theta}}$ & Weibull (1951) \\
\hline Exponential & - & 0 & 0 & 0 & 1 & $1-e^{-\alpha x}$ & Bain (1974) \\
\hline
\end{tabular}
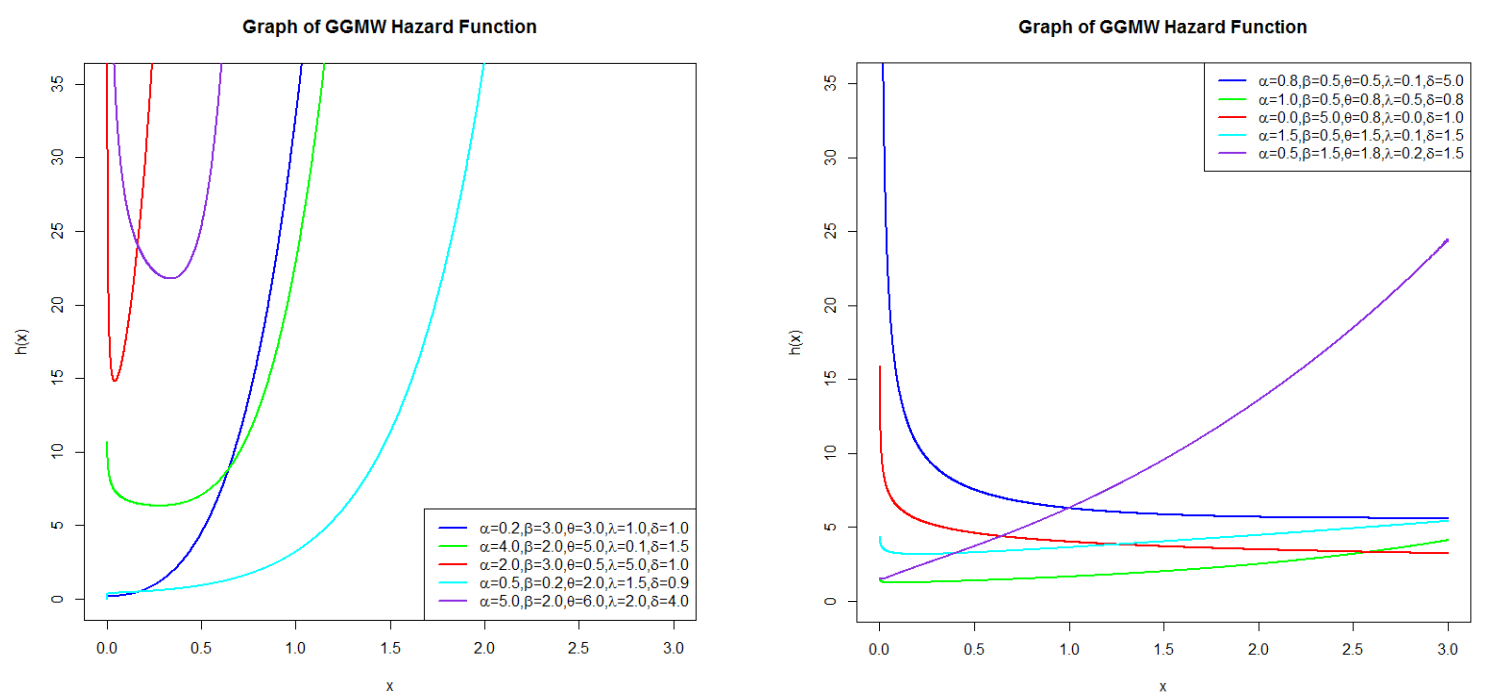

Figure 2: Graphs of GGMW hazard function 
$e^{-\beta x^{\theta} e^{\lambda x}}$ and $e^{k \lambda x}$, we have:

$$
\begin{aligned}
E\left(Y^{r}\right) & =\int_{0}^{\infty} y^{r} d\left(1-e^{-\alpha y-\beta x^{\theta} e^{\lambda y}}\right) \\
& =\int_{0}^{\infty} r y^{r-1} e^{-\alpha y-\beta y^{\theta} e^{\lambda y}} d y \\
& =\sum_{k, n=0}^{\infty} \frac{r(-\beta)^{n}(n \lambda)^{k}}{k ! n !} \int_{0}^{\infty} r y^{r+n \theta+k-1} e^{-\alpha y} d y \\
& =\sum_{k, n=0}^{\infty} \frac{r(-\beta)^{n}(n \lambda)^{k}}{k ! n !} \alpha^{-(r+\theta n+k)} \Gamma(r+\theta n+k) .
\end{aligned}
$$

Consequently, that the $r^{\text {th }}$ raw moment of GGMW distribution is given by:

$$
\mu_{r}^{\prime}=E\left(X^{r}\right)=\sum_{\nu \in C} w_{\nu} E\left(Y^{r}\right)
$$

where $Y \sim G M W(\alpha(m+s+\delta), \beta(m+s+\delta), \theta, \lambda)$. Note that, since $\sum_{r=0}^{\infty} \frac{t^{r}}{r !} x^{r} g_{G G M W}(x)$ converges and each term is integrable for all $t$ close to zero, say (for $|t|<1$ ), the moment generating function (MGF) of the GGMW distribution is given by:

$$
\begin{aligned}
M_{X}(t) & =\sum_{\nu \in C} \sum_{j=0}^{\infty} w_{\nu} \frac{t^{j}}{j !} E\left(Y^{j}\right) \\
& =\sum_{\nu \in C} \sum_{k, n, j=0}^{\infty} w_{\nu} \frac{t^{j} j(-\beta(k+s+\delta))^{n}(n \lambda)^{k}}{k ! n ! j !(\alpha(k+s+\delta))^{(j+\theta n+k)}} \Gamma(j+\theta n+k),
\end{aligned}
$$

where $\Gamma(a)=b^{a} \int_{0}^{\infty} t^{a-1} e^{-t} d t$ is the gamma function, and $r=1,2, \ldots \ldots$.

Table 3 lists the first six moments for selected parameter values of GGMW distribution, where Variance $=E\left(Y^{2}\right)-E(Y)^{2}$, Skewness $=\frac{E\left(Y^{3}\right)-3 E(Y) \sigma^{2}-E(Y)^{3}}{\sigma^{3}}$, and Kurtosis $=\frac{E\left(Y^{4}\right)}{\sigma^{4}}-3$.

Theorem 3.1.

$$
E\left\{[-\log (1-F(X))]^{r}\left[(1-F(X))^{s}\right]\right\}=\frac{\psi^{r+\delta} \Gamma(r+\delta)}{(s \psi+1)^{\delta} \psi^{\delta} \Gamma(\delta)} .
$$

\section{Proof:}

$$
\begin{aligned}
E\left\{[-\log (1-F(X))]^{r}\left[(1-F(X))^{s}\right]\right\} & =\int_{0}^{\infty} \frac{f(x)}{\psi^{\delta} \Gamma(\delta)}[-\log (1-F(x))]^{r+\delta-1} \\
& \times[1-F(x)]^{s+(1 / \psi)-1} d x \\
& =\frac{\psi^{r+\delta} \Gamma(r+\delta)}{(s \psi+1)^{\delta} \psi^{\delta} \Gamma(\delta)}
\end{aligned}
$$

If $s=0$ in equation (28), then we have

$$
E\left[-\log (1-F(X))^{r}\right]=\frac{\psi^{r+\delta} \Gamma(r+\delta)}{\psi^{\delta} \Gamma(\delta)} .
$$

Let $\psi^{*}=s+\frac{1}{\psi}$, then with $r=0$ in equation (28), we obtain

$$
\begin{aligned}
E\left[(1-F(X))^{s}\right] & =\left(\frac{1}{\psi \psi^{*}}\right)^{\delta} \int_{0}^{\infty} \frac{\left(\psi^{*}\right)^{\delta} f(x)}{\Gamma(\delta)}[-\log (1-F(x))]^{\delta-1} \\
& \times[1-F(x)]^{\psi^{*}-1} d x \\
& =[s \psi+1]^{-\delta} .
\end{aligned}
$$


Table 3: GGMW moments for selected values

\begin{tabular}{cccccc}
\hline & \multicolumn{5}{c}{$(\alpha, \beta, \theta, \lambda, \delta)$} \\
\cline { 2 - 6 } Moments & $(1,2,0.5,0.5,1)$ & $(1,2,0.5,1.5,2)$ & $(1,4,2,1,6)$ & $(1,1.5,2,1,2.5)$ & $(2,0.9,1,1,3)$ \\
\hline$E(Y)$ & 0.1798084 & 0.0360182 & 0.0130699 & 0.1442460 & 0.0502597 \\
$E\left(Y^{2}\right)$ & 0.0883142 & 0.0050822 & 0.0007539 & 0.0394158 & 0.0071370 \\
$E\left(Y^{3}\right)$ & 0.0649863 & 0.0011492 & 0.0000767 & 0.0142427 & 0.0016577 \\
$E\left(Y^{4}\right)$ & 0.0608936 & 0.0003385 & 0.0000107 & 0.0060942 & 0.0005210 \\
$E\left(Y^{5}\right)$ & 0.0674815 & 0.0001191 & 0.0000018 & 0.0029347 & 0.0002021 \\
$E\left(Y^{6}\right)$ & 0.0848496 & 0.0000477 & 0.0000004 & 0.0015457 & 0.0000917 \\
Variance & 0.0559832 & 0.0037849 & 0.0005831 & 0.0186089 & 0.0046110 \\
Skewness & 2.1873821 & 2.9781690 & 3.6632310 & 1.2561066 & 2.6683440 \\
Kurtosis & 16.4292658 & 20.6287000 & 28.4321500 & 14.5986436 & 21.5064600 \\
\hline
\end{tabular}

\section{Order statistics and Rényi entropy}

Order statistics play an important role in probability and statistics. The concept of entropy plays a vital role in information theory. The entropy of a random variable is defined in terms of its probability distribution and can be shown to be a good measure of randomness or uncertainty. In this section, we present Rényi entropy and the distribution of the order statistics for the GGMW distribution.

\subsection{Rényi entropy}

Rényi entropy is an extension of Shannon entropy. Rényi entropy is defined to be

$$
I_{R}(v)=\frac{1}{1-v} \log \left(\int_{0}^{\infty}\left[g_{G G M W}(x ; \alpha, \beta, \theta, \lambda, \delta)\right]^{v} d x\right), v \neq 1, v>0 .
$$

Rényi entropy tends to Shannon entropy as $v \rightarrow 1$. Note that

$$
\begin{aligned}
\int_{0}^{\infty} g_{G G M W}^{v}(x) d x & =\left(\frac{1}{\Gamma(\delta)}\right)^{v} \int_{0}^{\infty}\left(\left(\alpha+\beta x^{\theta-1} e^{\lambda x}[\theta+\lambda x]\right) e^{-\alpha x-\beta x^{\theta} e^{\lambda x}}\right)^{v} \\
& \times\left[-\log \left(1-e^{-\alpha x-\beta x^{\theta} e^{\lambda x}}\right)\right]^{v(\delta-1)} d x
\end{aligned}
$$

Let $0<y=e^{-\alpha x-\beta x^{\theta} e^{\lambda x}}<0.7968$. Note that

$$
\begin{aligned}
\left(\left(\alpha+\beta x^{\theta-1} e^{\lambda x}[\theta+\lambda x]\right)\right)^{v} & =\sum_{j=0}^{v}\left(\begin{array}{l}
v \\
j
\end{array}\right) \alpha^{v-j} \beta^{j} x^{j \theta-j} \sum_{n=0}^{\infty}\left(\begin{array}{l}
j \\
r
\end{array}\right) \frac{(j \lambda x)^{n}}{n !} \sum_{r=0}^{j} \theta^{j-r}(\lambda x)^{r} \\
& =\sum_{j=0}^{v} \sum_{r=0}^{j} \sum_{n=0}^{\infty}\left(\begin{array}{l}
v \\
j
\end{array}\right)\left(\begin{array}{l}
j \\
r
\end{array}\right) \alpha^{v-j} \beta^{j} \theta^{j-r} \lambda^{r} \frac{(j \lambda)^{n}}{n !} x^{n+r+j \theta-j}
\end{aligned}
$$

Now, for $0<e^{-v \beta x^{\theta} e^{\lambda x}}<1, v>0$, and applying Taylor series expansion, we have

$$
e^{-v \beta x^{\theta} e^{\lambda x}}=\sum_{l=0}^{\infty} \sum_{w=0}^{\infty} \frac{(-1)^{l}(v \beta)^{l}(l \lambda)^{w}}{l ! w !} x^{l \theta+w},
$$


so that,

$$
\begin{aligned}
& g^{v}(x)=[\Gamma(\delta)]^{-v} \sum_{j=0}^{v} \sum_{r=0}^{j} \sum_{n, l, w, m, s=0}^{\infty}(-1)^{l}\left(\begin{array}{l}
v \\
j
\end{array}\right)\left(\begin{array}{l}
j \\
r
\end{array}\right)\left(\begin{array}{c}
\delta(v-1) \\
m
\end{array}\right) \\
& \times \quad \alpha^{v-j} \beta^{j} \theta^{j-r} \lambda^{r} \frac{(j \lambda)^{n}}{n !} \frac{(v \beta)^{l}}{l !} \frac{(l \lambda)^{w}}{w !} b_{s, m} \\
& \times \quad x^{n+r+j \theta-j+l \theta+w} e^{-(m+s+v \delta-v) \alpha x-(m+s+v \delta-v) \beta x^{\theta} e^{\lambda x}} e^{-v \alpha x} \\
& =[\Gamma(\delta)]^{-v} \sum_{j=0}^{v} \sum_{r=0}^{j} \sum_{n, l, w, m, s, k, i=0}^{\infty}(-1)^{l+k}\left(\begin{array}{l}
v \\
j
\end{array}\right)\left(\begin{array}{l}
j \\
r
\end{array}\right)\left(\begin{array}{c}
\delta(v-1) \\
m
\end{array}\right) b_{s, m} \\
& \times \quad \alpha^{v-j} \beta^{j+l} \theta^{j-r} \lambda^{r+n+w} \frac{(j)^{n}(v)^{l}(l)^{w}}{n ! l ! w !} \\
& \times \quad \frac{(m+s+v \delta-v)^{k} \beta^{k}(k \lambda)^{i}}{k ! i !} x^{n+r+j \theta-j+l \theta+w+k \theta+i} e^{-(m+s+v \delta) \alpha x} .
\end{aligned}
$$

Using the fact that $\int_{0}^{\infty} t^{a-1} e^{-t} d t=\frac{\Gamma(a)}{b^{a}}$, we have

$$
\begin{aligned}
\int_{0}^{\infty} g_{G G M W}^{v}(x) d x & =[\Gamma(\delta)]^{-v} \sum_{j=0}^{v} \sum_{r=0}^{j} \sum_{n, l, w, m, s, k, i=0}^{\infty}(-1)^{l+k}\left(\begin{array}{l}
v \\
j
\end{array}\right)\left(\begin{array}{l}
j \\
r
\end{array}\right)\left(\begin{array}{c}
\delta(v-1) \\
m
\end{array}\right) b_{s, m} \\
& \times \alpha^{v-j} \beta^{j+l+} \theta^{j-r} \lambda^{r+n+w+i} \frac{(j)^{n}(v)^{l}(l)^{w} k^{i}(m+s+v \delta-v)^{k}}{n ! l ! w ! k ! i !} \\
& \times \frac{\Gamma(n+r+w+i+\theta(j+l+k)-j+1)}{(m+s+v \delta)^{n+r+w+i+\theta(j+l+k)-j+1}}
\end{aligned}
$$

for $v>0, v \neq 1$. Consequently, Rényi entropy for the GGMW distribution is given by

$$
\begin{aligned}
I_{R}(v) & =\frac{1}{1-v} \log \left[[\Gamma(\delta)]^{-v} \sum_{j=0}^{v} \sum_{r=0}^{j} \sum_{n, l, w, m, s, k, i=0}^{\infty}(-1)^{l+k}\left(\begin{array}{l}
v \\
j
\end{array}\right)\left(\begin{array}{l}
j \\
r
\end{array}\right)\left(\begin{array}{c}
\delta(v-1) \\
m
\end{array}\right) b_{s, m}\right. \\
& \times \alpha^{v-j} \beta^{j+l+} \theta^{j-r} \lambda^{r+n+w+i} \frac{(j)^{n}(v)^{l}(l)^{w} k^{i}(m+s+v \delta-v)^{k}}{n ! l ! w ! k ! i !} \\
& \left.\times \frac{\Gamma(n+r+w+i+\theta(j+l+k)-j+1)}{(m+s+v \delta)^{n+r+w+i+\theta(j+l+k)-j+1}}\right], \quad \text { for } v>0, v \neq 1 .
\end{aligned}
$$

\subsection{Order statistics}

In this section, the pdf of the $i^{t h}$ order statistic and the corresponding moments are presented. Let $X_{1}, X_{2}, \ldots, X_{n}$ be independent and identically distributed GGMW random variables. The pdf of of the $i^{\text {th }}$ order statistic for a random sample of size $n$ for any gamma $-\bar{G}$ family with density (5) can be expressed as an infinite weighted sum of gamma $-\bar{G}$ densities. The pdf of the $i^{t h}$ order statistic from the GGMW pdf $g_{G G M W}(x)$ is given by

$$
\begin{aligned}
g_{i: n}(x) & =\frac{n ! g(x)}{(i-1) !(n-i) !}[G(x)]^{i-1}[1-G(x)]^{n-i} \\
& =\frac{n ! g(x)}{(i-1) !(n-i) !} \sum_{j=0}^{i-1}(-1)^{j}\left(\begin{array}{c}
i-1 \\
j
\end{array}\right)[\bar{G}(x)]^{n-i+j} \\
& =\frac{n ! g(x)}{(i-1) !(n-i) !} \sum_{j=0}^{i-1}(-1)^{j}\left(\begin{array}{c}
i-1 \\
j
\end{array}\right)\left[\frac{\gamma\left(-\log \left(1-e^{-\alpha x-\beta x^{\theta} e^{\lambda x}}\right)\right)}{\Gamma(\delta)}\right]^{n-i+j} .
\end{aligned}
$$

where $0<y=e^{-\alpha x-\beta x^{\theta} e^{\lambda x}}<0.7968, x>0, \alpha, \beta, \theta, \delta>0$, and $\lambda \geq 0$. Using the fact that $\gamma(x, \delta)=\sum_{m=0}^{\infty} \frac{(-1)^{m} x^{m+\delta}}{(m+\delta) m !}$, and setting $c_{m}=(-1)^{m} /((m+\delta) m !)$, we can write the pdf of the 
$i^{\text {th }}$ order statistic from the GGMW distribution as follows:

$$
\begin{aligned}
g_{i: n}(x) & =\frac{n ! g(x)}{(i-1) !(n-i) !} \sum_{j=0}^{i-1}\left(\begin{array}{c}
i-1 \\
j
\end{array}\right) \frac{(-1)^{j}}{[\Gamma(\delta)]^{n-i+j}}\left[-\log \left(1-e^{\left.\left.-\alpha x-\beta x^{\theta} e^{\lambda x}\right)\right]^{\delta(n-i+j)}}\right.\right. \\
& \times\left[\sum_{m=0}^{\infty} \frac{(-1)^{m}\left(\operatorname { l o g } \left(1-e^{\left.\left.-\alpha x-\beta x^{\theta} e^{\lambda x}\right)\right)^{m}}\right.\right.}{(m+\delta) m !}\right]^{n-i+j} \\
& =\frac{n ! g(x)}{(i-1) !(n-i) !} \sum_{j=0}^{i-1}\left(\begin{array}{c}
i-1 \\
j
\end{array}\right) \frac{(-1)^{j}}{[\Gamma(\delta)]^{n-i+j}}\left[-\log \left(1-e^{-\alpha x-\beta x^{\theta} e^{\lambda x}}\right)\right]^{\delta(n-i+j)} \\
& \times \sum_{m=0}^{\infty} d_{m, n-i+j}\left(-\log \left(1-e^{-\alpha x-\beta x^{\theta} e^{\lambda x}}\right)\right)^{m},
\end{aligned}
$$

where $d_{0}=c_{0}^{(n-i+j)}, d_{m, n-i+j}=\left(m c_{0}\right)^{-1} \sum_{l=1}^{m}[(n-i+j) l-m+l] c_{l} d_{m-l, n-i+j}$. We note that

$$
\begin{aligned}
& g_{i: n}(x)=\frac{n ! g(x)}{(i-1) !(n-i) !} \sum_{j=0}^{i-1} \sum_{m=0}^{\infty}\left(\begin{array}{c}
n-i \\
j
\end{array}\right) \frac{(-1)^{j} d_{m, i+j-1}}{[\Gamma(\delta)]^{n-i+j}}\left[-\log \left(1-e^{-\alpha x-\beta x^{\theta} e^{\lambda x}}\right)\right]^{\delta(n-i+j)+m} \\
& =\frac{n !\left[-\log \left(1-e^{-\alpha x-\beta x^{\theta} e^{\lambda x}}\right)\right]^{\delta-1} f(x)}{(i-1) !(n-i) ! \Gamma(\delta)} \sum_{j=0}^{i-1} \sum_{m=0}^{\infty}\left(\begin{array}{c}
n-i \\
j
\end{array}\right) \frac{(-1)^{j} d_{m, n-i+j}}{[\Gamma(\delta)]^{n-i+j}} \\
& \times \quad\left[-\log \left(1-e^{-\alpha x-\beta x^{\theta} e^{\lambda x}}\right)\right]^{\delta(n-i+j)+m} \\
& =\frac{n !}{(i-1) !(n-i) !} \sum_{j=0}^{i-1} \sum_{m=0}^{\infty}\left(\begin{array}{c}
i-1 \\
j
\end{array}\right) \frac{(-1)^{j} d_{m, n-i+j}}{[\Gamma(\delta)]^{n-i+j}} \\
& \times \frac{\Gamma(\delta(n-i+j)+m+\delta)}{\Gamma(\delta(n-i+j)+m+\delta)} \frac{\left[-\log \left(1-e^{\left.\left.-\alpha x-\beta x^{\theta} e^{\lambda x}\right)\right]^{\delta(n-i+j)+m+\delta-1}}\right.\right.}{\Gamma(\delta)} \\
& \times\left(\alpha+\beta x^{\theta-1} e^{\lambda x}[\theta+\lambda x]\right) e^{-\alpha x-\beta x^{\theta} e^{\lambda x}} \\
& =\frac{n !}{(i-1) !(n-i) !} \sum_{j=0}^{i-1} \sum_{m=0}^{\infty}\left(\begin{array}{c}
i-1 \\
j
\end{array}\right) \\
& \times \frac{(-1)^{j} d_{m, n-i+j} \Gamma(\delta(n-i+j)+m+\delta)}{[\Gamma(\delta)]^{n-i+j+1}} f_{G G M W}(x),
\end{aligned}
$$

where

$$
\begin{aligned}
f_{G G M W}(x) & =\frac{1}{\Gamma(\delta(n-i+j)+m+\delta)}\left[-\log \left(1-e^{-\alpha x-\beta x^{\theta} e^{\lambda x}}\right)\right]^{\delta(n-i+j)+m+\delta-1} \\
& \times\left(\alpha+\beta x^{\theta-1} e^{\lambda x}[\theta+\lambda x]\right) e^{-\alpha x-\beta x^{\theta} e^{\lambda x}}
\end{aligned}
$$

is the GGMW pdf with parameters $\alpha, \beta, \theta>0, \lambda \geq 0$, and shape parameter $\delta^{*}=\delta(n-i+$ $j)+m+\delta>0$. It follows therefore that the $r^{t h}$ moment is given by

$$
E\left(X_{i: n}^{j}\right)=\sum_{\nu \in C} \sum_{j=0}^{i-1} \sum_{m, k, n=0}^{\infty} w_{\nu} \ell_{i, j, m} \frac{r(-\beta)^{n}\left(k+s+\delta^{*}\right)(n \lambda)^{k}}{k ! n !\left[\alpha\left(k+s+\delta^{*}\right)^{r+n \theta+k}\right]} \Gamma(r+n \theta+k),
$$

where $\ell_{i, j, m}=\frac{n !}{(i-1) !(n-i) !} \frac{(-1)^{j} d_{m, n-i+j} \Gamma(\delta(n-i+j)+m+\delta)}{[\Gamma(\delta)]^{n-i+j+1}}$, and $\delta^{*}=\delta(n-i+j)+m+\delta>0$. We note that these moments are often used in several areas including reliability, survival analysis, biometry, engineering, insurance and quality control for the prediction of future failures times from a set of past or previous failures. 


\section{Maximum likelihood estimation}

Let $X \sim G G M W(\alpha, \beta, \theta, \lambda, \delta)$ and $\boldsymbol{\Delta}=(\alpha, \beta, \theta, \lambda, \delta)^{T}$ be the parameter vector. The loglikelihood for a single observation $x$ of $X$ is given by

$$
\begin{aligned}
\ell=\ell(\boldsymbol{\Delta}) & =(\delta-1) \log \left(-\log \left(1-e^{-\alpha x-\beta x^{\theta} e^{\lambda x}}\right)\right)+\log \left(\alpha+\beta x^{\theta-1} e^{\lambda x}[\theta+\lambda x]\right) \\
& -\alpha x-\beta x^{\theta} e^{\lambda x}-\log (\Gamma(\delta)) .
\end{aligned}
$$

The first derivative of the log-likelihood function with respect to the parameters $\boldsymbol{\Delta}=(\alpha, \beta, \theta, \lambda, \delta)^{T}$ are given by

$$
\begin{aligned}
& \frac{\partial \ell}{\partial \alpha}=\frac{x(\delta-1) e^{-\alpha x-\beta x^{\theta} e^{\lambda x}}}{\left(1-e^{-\alpha x-\beta x^{\theta} e^{\lambda x}}\right) \log \left(1-e^{-\alpha x-\beta x^{\theta} e^{\lambda x}}\right)}+\frac{1}{\alpha+\beta x^{\theta-1} e^{\lambda x}[\theta+\lambda x]}-x, \\
& \frac{\partial \ell}{\partial \beta}=\frac{x^{\theta} e^{\lambda x}(\delta-1) e^{-\alpha x-\beta x^{\theta} e^{\lambda x}}}{\left(1-e^{-\alpha x-\beta x^{\theta} e^{\lambda x}}\right) \log \left(1-e^{-\alpha x-\beta x^{\theta} e^{\lambda x}}\right)}+\frac{x^{\theta-1} e^{\lambda x}(\theta+\lambda x)}{\alpha+\beta x^{\theta-1} e^{\lambda x}[\theta+\lambda x]}-x^{\theta} e^{\lambda x}, \\
& \frac{\partial \ell}{\partial \theta}=\frac{(\delta-1) x^{\theta} \log (x) \beta e^{\lambda x} e^{-\alpha x-\beta x^{\theta} e^{\lambda x}}}{\left(1-e^{-\alpha x-\beta x^{\theta} e^{\lambda x}}\right) \log \left(1-e^{-\alpha x-\beta x^{\theta} e^{\lambda x}}\right)}+\frac{\beta x^{\theta-1} e^{\lambda x}[(\theta+\lambda x) \log (x)+1]}{\alpha+\beta x^{\theta-1} e^{\lambda x}[\theta+\lambda x]} \\
& -\beta x^{\theta} e^{\lambda x} \log (x) \\
& \frac{\partial \ell}{\partial \lambda}=\frac{(\delta-1) x^{\theta+1} \beta e^{\lambda x} e^{-\alpha x-\beta x^{\theta} e^{\lambda x}}}{\left(1-e^{-\alpha x-\beta x^{\theta} e^{\lambda x}}\right) \log \left(1-e^{-\alpha x-\beta x^{\theta} e^{\lambda x}}\right)}+\frac{\beta x^{\theta} e^{\lambda x}(\theta+\lambda x+1)}{\alpha+\beta x^{\theta-1} e^{\lambda x}[\theta+\lambda x]}-\beta x^{\theta+1} e^{\lambda x},
\end{aligned}
$$

and

$$
\frac{\partial \ell}{\partial \delta}=\log \left(-\log \left(1-e^{-\alpha x-\beta x^{\theta} e^{\lambda x}}\right)\right)-\frac{\Gamma^{\prime}(\delta)}{\Gamma(\delta)} .
$$

The total log-likelihood function based on a random sample of $n$ observations: $x_{1}, x_{2}, \ldots, x_{n}$ drawn from the GGMW distribution is given by $\ell_{n}=\ell(\boldsymbol{\Delta})=\sum_{i=1}^{n} \ell_{i}(\boldsymbol{\Delta})$, where $\ell_{i}(\boldsymbol{\Delta})$, $i=1,2, \ldots ., n$ is given by equation (35). The equations obtained by setting the above partial derivatives to zero are not in closed form and the values of the parameters $\alpha, \beta, \theta, \lambda, \delta$ must be found by using iterative methods. The maximum likelihood estimates of the parameters, denoted by $\hat{\boldsymbol{\Delta}}$ is obtained by solving the nonlinear equations $\left(\frac{\partial \ell}{\partial \alpha}, \frac{\partial \ell}{\partial \beta}, \frac{\partial \ell}{\partial \theta}, \frac{\partial \ell}{\partial \lambda}, \frac{\partial \ell}{\partial \delta}\right)^{T}=\mathbf{0}$. It is convenient to apply or use nonlinear optimization algorithm such as quasi-Newton algorithm to numerically maximize the log-likelihood function.

We maximize the likelihood function using NLmixed in SAS as well as the function nlm in R (The R Development Core Team (2011)). These functions were applied and executed for wide range of initial values. This process often results or lead to more than one maximum, however, in these cases, we take the MLEs corresponding to the largest value of the maxima. In a few cases, no maximum was identified for the selected initial values. In these cases, a new initial value was tried in order to obtain a maximum.

The issues of existence and uniqueness of the MLEs are theoretical interest and has been studied by several authors for different distributions including Seregin (2010), Santos Silva and Tenreyro (2010), Zhou (2009), and Xia, Mi, and Zhou (2009). At this point we are not able to address the theoretical aspects (existence, uniqueness) of the MLE of the parameters of the GGMW distribution.

Note that for the five parameters of the GGMW distribution, all second order partial derivatives of the log-likelihood function exit, and are given in appendix A. The Fisher information matrix is given by $\mathbf{I}(\boldsymbol{\Delta})=\left[\mathbf{I}_{\theta_{i}, \theta_{j}}\right]_{5 X 5}=E\left(-\frac{\partial^{2} \ell}{\partial \theta_{i} \partial \theta_{j}}\right), i, j=1,2,3,4,5$, can be numerically 
obtained by MATHLAB, R or MAPLE software. The total Fisher information matrix $n \mathbf{I}(\boldsymbol{\Delta})$ can be approximated by

$$
\mathbf{J}_{n}(\hat{\boldsymbol{\Delta}}) \approx\left[-\left.\frac{\partial^{2} \ell}{\partial \theta_{i} \partial \theta_{j}}\right|_{\boldsymbol{\Delta}=\hat{\boldsymbol{\Delta}}}\right]_{5 X 5}, \quad i, j=1,2,3,4,5 .
$$

For a given set of observations, the matrix given in equation (41) is obtained after the convergence of the Newton-Raphson procedure in MATHLAB or R software. Elements of the observed information matrix are given in the appendix.

\subsection{Asymptotic confidence intervals}

In this section, we present the asymptotic confidence intervals for the parameters of the GGMW distribution. The expectations in the Fisher Information Matrix (FIM) can be obtained numerically. Let $\hat{\boldsymbol{\Delta}}=(\hat{\alpha}, \hat{\beta}, \hat{\theta}, \hat{\lambda}, \hat{\delta})$ be the maximum likelihood estimate of $\boldsymbol{\Delta}=$ $(\alpha, \beta, \theta, \lambda, \delta)$. Under the usual regularity conditions and that the parameters are in the interior of the parameter space, but not on the boundary, (Ferguson 1996) we have: $\sqrt{n}(\hat{\boldsymbol{\Delta}}-\boldsymbol{\Delta}) \stackrel{d}{\longrightarrow}$ $N_{5}\left(\underline{0}, I^{-1}(\boldsymbol{\Delta})\right)$, where $I(\boldsymbol{\Delta})$ is the expected Fisher information matrix. The asymptotic behavior is still valid if $I(\boldsymbol{\Delta})$ is replaced by the observed information matrix evaluated at $\hat{\boldsymbol{\Delta}}$, that is $J(\hat{\boldsymbol{\Delta}})$. The multivariate normal distribution $N_{5}\left(\underline{\mathbf{0}}, J(\hat{\boldsymbol{\Delta}})^{-1}\right)$, where the mean vector $\underline{\mathbf{0}}=(0,0,0,0,0)^{T}$, can be used to construct confidence intervals and confidence regions for the individual model parameters and for the survival and hazard rate functions. That is, the approximate $100(1-\eta) \%$ two-sided confidence intervals for $\alpha, \beta, \theta \lambda$, and $\delta$ are given by:

$$
\widehat{\alpha} \pm Z_{\frac{\eta}{2}} \sqrt{I_{\alpha \alpha}^{-1}(\hat{\boldsymbol{\Delta}})}, \quad \widehat{\beta} \pm Z_{\frac{\eta}{2}} \sqrt{I_{\beta \beta}^{-1}(\hat{\boldsymbol{\Delta}})}, \quad \widehat{\theta} \pm Z_{\frac{\eta}{2}} \sqrt{I_{\theta \theta}^{-1}(\hat{\boldsymbol{\Delta}})}, \quad \widehat{\lambda} \pm Z_{\frac{\eta}{2}} \sqrt{I_{\lambda \lambda}^{-1}(\hat{\boldsymbol{\Delta}})},
$$

and $\widehat{\delta} \pm Z_{\frac{\eta}{2}} \sqrt{I_{\delta \delta}^{-1}(\hat{\boldsymbol{\Delta}})}$, respectively, where $I_{\alpha \alpha}^{-1}(\hat{\boldsymbol{\Delta}}), I_{\beta \beta}^{-1}(\hat{\boldsymbol{\Delta}}), I_{\theta \theta}^{-1}(\hat{\boldsymbol{\Delta}}), I_{\lambda \lambda}^{-1}(\hat{\boldsymbol{\Delta}})$ and $I_{\delta \delta}^{-1}(\hat{\boldsymbol{\Delta}})$ are the diagonal elements of $I_{n}^{-1}(\hat{\boldsymbol{\Delta}})$, and $Z_{\frac{\eta}{2}}$ is the upper $\frac{\eta}{2}^{t h}$ percentile of a standard normal distribution.

The maximum likelihood estimates (MLEs) of the GGMW parameters $\alpha, \beta, \theta, \lambda$, and $\delta$ are computed by maximizing the objective function via the subroutine NLmixed in SAS and the function $\mathrm{nlm}$ in $\mathrm{R}$. The estimated values of the parameters (standard error in parenthesis), -2log-likelihood statistic, Akaike Information Criterion, $A I C=2 p-2 \ln (L)$, Bayesian Information Criterion, $B I C=p \ln (n)-2 \ln (L)$, and Consistent Akaike Information Criterion, $A I C C=A I C+2 \frac{p(p+1)}{n-p-1}$, where $L=L(\hat{\boldsymbol{\Delta}})$ is the value of the likelihood function evaluated at the parameter estimates, $n$ is the number of observations, and $p$ is the number of estimated parameters are presented. In order to compare the models, we use the criteria stated above. Note that for the value of the log-likelihood function at its maximum $\left(\ell_{n}\right)$, larger value is good and preferred, and for AIC, AICC and BIC, smaller values are preferred. GGMW distribution is fitted to the data sets and these fits are compared to the fits of the GGME, GGMR, GMW, GW, beta exponentiated Weibull (BEW) and beta Weibull (BW) distributions.

We can use the likelihood ratio (LR) test to compare the fit of the GGMW distribution with its sub-models for a given data set. For example, to test $\lambda=0, \delta=1$, the LR statistic is $\omega=2[\ln (L(\hat{\alpha}, \hat{\beta}, \hat{\theta}, \hat{\lambda}, \hat{\delta}))-\ln (L(\tilde{\alpha}, \tilde{\beta}, \tilde{\theta}, 0,1))]$, where $\hat{\alpha}, \hat{\beta}, \hat{\lambda}, \hat{\theta}$ and $\hat{\delta}$, are the unrestricted estimates, and $\tilde{\alpha}, \tilde{\beta}$, and $\tilde{\theta}$ are the restricted estimates. The LR test rejects the null hypothesis if $\omega>\chi_{\epsilon}^{2}$, where $\chi_{\epsilon}^{2}$ denote the upper $100 \epsilon \%$ point of the $\chi^{2}$ distribution with 2 degrees of freedom.

\section{Applications}

In this section, we present examples to illustrate the flexibility and applicability of the GGMW distribution and its sub-models for data modeling. The GGMW distribution is also compared 
Table 4: Estimation of GGMW model for waiting times data

\begin{tabular}{|c|c|c|c|c|c|c|c|c|c|c|}
\hline \multirow[b]{2}{*}{ Distribution } & \multicolumn{4}{|c|}{ Estimates } & \multicolumn{5}{|c|}{ Statistics } & \multirow[b]{2}{*}{ SS } \\
\hline & $\alpha$ & $\beta$ & $\theta$ & $\lambda$ & $\delta$ & -2LogLikelihood & AIC & AICC & BIC & \\
\hline GGMW & $\begin{array}{c}0.3529 \\
(0.01679)\end{array}$ & $\begin{array}{c}0.05611 \\
(0.02513)\end{array}$ & $\begin{array}{c}1.6133 \\
(0.1642)\end{array}$ & $\begin{array}{c}0.002399 \\
(0.01078)\end{array}$ & $\begin{array}{c}0.1687 \\
(0.01386)\end{array}$ & 637.7 & 647.7 & 648.3 & 660.7 & 0.0574 \\
\hline GGME & $\begin{array}{c}0.000223 \\
(0.292)\end{array}$ & $\begin{array}{c}0.4152 \\
(0.2142)\end{array}$ & 1 & $\begin{array}{c}0.02139 \\
(0.006622)\end{array}$ & $\begin{array}{c}0.1875 \\
(0.02897)\end{array}$ & 640.5 & 648.5 & 649 & 659 & 0.0929 \\
\hline GMW & 0 & $\begin{array}{c}0.2887 \\
(0.07322)\end{array}$ & $\begin{array}{c}1.3239 \\
(0.1062)\end{array}$ & $\begin{array}{c}0.000107 \\
(0.005437)\end{array}$ & $\begin{array}{c}0.1629 \\
(0.01887)\end{array}$ & 634.8 & 642.8 & 643.2 & 653.2 & 0.0271 \\
\hline GME & 0 & $\begin{array}{c}0.3892 \\
(0.09978)\end{array}$ & 1 & $\begin{array}{c}0.01897 \\
(0.006636)\end{array}$ & $\begin{array}{c}0.2058 \\
(0.03738)\end{array}$ & 640.9 & 646.9 & 647.2 & 654.7 & 0.1024 \\
\hline GAE & $\begin{array}{c}0.2108 \\
(0.09663)\end{array}$ & $\begin{array}{c}0.1808 \\
(0.09657)\end{array}$ & 1 & 0 & $\begin{array}{c}0.2776 \\
(0.141)\end{array}$ & 647.9 & 653.9 & 654.1 & 661.7 & 0.3585 \\
\hline GEV & 0 & 1 & 0 & $\begin{array}{c}0.09372 \\
(0.000906)\end{array}$ & $\begin{array}{c}0.3111 \\
(0.02987)\end{array}$ & 727.1 & 731.1 & 731.2 & 736.3 & 1.1924 \\
\hline GW & 0 & $\begin{array}{c}0.2443 \\
(0.07656)\end{array}$ & $\begin{array}{c}1.3435 \\
(0.08978)\end{array}$ & 0 & $\begin{array}{c}0.1829 \\
(0.0236)\end{array}$ & 635.3 & 641.3 & 641.6 & 649.2 & 0.0329 \\
\hline BW & $\begin{array}{c}k \\
1.2455 \\
(0.1008)\end{array}$ & $\begin{array}{c}\lambda \\
2.1348 \\
(0.4812)\end{array}$ & $\begin{array}{c}a \\
1.7298 \\
(0.5264)\end{array}$ & $\begin{array}{c}b \\
0.1509 \\
(0.01871)\end{array}$ & & 634.2 & 642.2 & 642.7 & 652.7 & 0.0239 \\
\hline BEW & $\begin{array}{c}k \\
1.135 \\
(0.2224)\end{array}$ & $\begin{array}{c}\lambda \\
2.6752 \\
(1.4164)\end{array}$ & $\begin{array}{c}\alpha \\
1.6422 \\
(1.3948)\end{array}$ & $\begin{array}{c}a \\
1.3894 \\
(0.7837)\end{array}$ & $\begin{array}{c}b \\
0.2681 \\
(0.2345)\end{array}$ & 633.9 & 643.9 & 644.6 & 657 & 0.0159 \\
\hline
\end{tabular}

with the non-nested beta exponentiated Weibull (BEW), and beta Weibull (BW) distributions. The pdf of the BEW distribution (Cordeiro, Gomes, da Silva, and Ortega 2013) is given by

$$
g(x)=\frac{\alpha k \lambda^{k}}{B(a, b)} x^{k-1} e^{(\lambda x)^{k}}\left(1-e^{(\lambda x)^{k}}\right)^{a \alpha-1}\left[1-\left(1-e^{(\lambda x)^{k}}\right)^{\alpha}\right]^{b-1}, \quad x>0 .
$$

When $\alpha=1$, we have the BW distribution.

The first data set is waiting times (in minutes) of 100 bank customers before service. See (Ghitany, Atieh, and Nadarajah 2008) for additional details. The second data set is failure times of a sample of $n=30$ devices, see (Meeker and Escobar 1998). The third data set represent the survival times of 121 patients with breast cancer obtained from a large hospital in a period from 1929 to 1938, (Lee 1992).

Estimates of the parameters of GGMW distribution (standard error in parentheses), Akaike Information Criterion (AIC), Consistent Akaike Information Criterion (AICC) and Bayesian Information Criterion (BIC) are given in Table 4 for the first data set, in Table 5 for the second data set and in Table 6 for the third data set.

The estimated covariance matrix for the GGMW distribution (Waiting Times Data) is given by

$$
\left(\begin{array}{ccccc}
0.00028 & -0.00110 & 0.00300 & 0.00020 & 0.00054 \\
-0.00110 & 0.00063 & -0.00294 & 0.00004 & -0.00001 \\
0.00300 & -0.00294 & 0.02697 & -0.00141 & -0.00048 \\
0.00020 & 0.00004 & -0.00141 & 0.00012 & 0.00003 \\
0.00054 & -0.00001 & -0.00048 & 0.00003 & 0.00019
\end{array}\right)
$$

The $95 \%$ asymptotic confidence intervals for the GGMW model (Waiting Times Data) parameters are: $\alpha \in(0.3200,0.3529), \beta \in(0.0069,0.1054), \theta \in(1.2915,1.9351), \lambda \in(-0.0187,0.0235)$, and $\delta \in(0.1415,0.1959)$, respectively.

The estimated covariance matrix for the GGMW distribution (Meeker Data) is given by

$$
\left(\begin{array}{ccccc}
0.000015 & -0.000010 & 0.000399 & 0.000000 & -0.000001 \\
-0.000010 & 0.000008 & -0.000040 & -0.000005 & 0.000002 \\
0.000399 & -0.000040 & 0.007974 & -0.000140 & -0.000110 \\
0.000000 & -0.000005 & -0.000140 & 0.000007 & 0.000001 \\
-0.000001 & 0.000002 & -0.000110 & 0.000001 & 0.000145
\end{array}\right)
$$


Fitted PDF

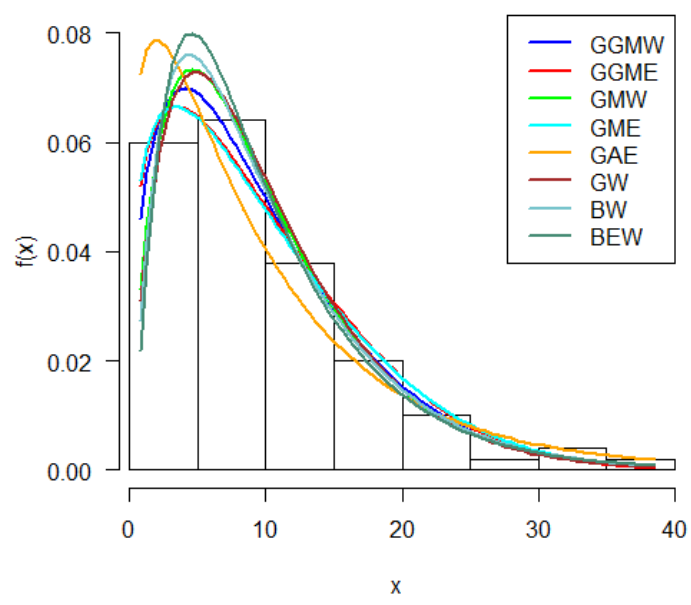

The Graph of Observed vs Expected Probability

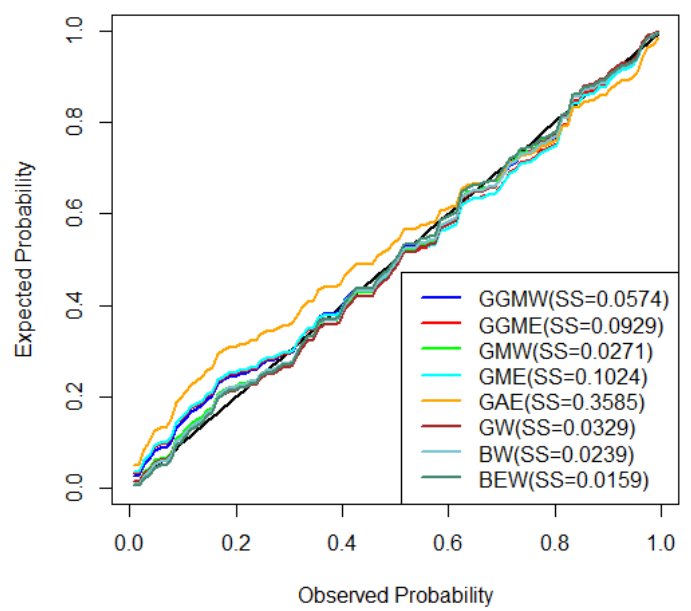

Figure 3: Graphs for waiting times data

Table 5: Estimation of GGMW model for meeker data

\begin{tabular}{|c|c|c|c|c|c|c|c|c|c|c|}
\hline \multirow[b]{2}{*}{ Distribution } & \multicolumn{4}{|c|}{ Estimates } & \multicolumn{5}{|c|}{ Statistics } & \multirow[b]{2}{*}{ SS } \\
\hline & $\alpha$ & $\beta$ & $\theta$ & $\lambda$ & $\delta$ & -2LogLikelihood & AIC & AICC & $\mathrm{BIC}$ & \\
\hline GGMW & $\begin{array}{c}0.05354 \\
(0.00392)\end{array}$ & $\begin{array}{c}0.004011 \\
(0.002771)\end{array}$ & $\begin{array}{c}0.004549 \\
(0.0893)\end{array}$ & $\begin{array}{c}0.02772 \\
(0.002629)\end{array}$ & $\begin{array}{c}0.06625 \\
(0.01203)\end{array}$ & 345.2 & 355.2 & 357.7 & 362.2 & 0.1885 \\
\hline GGME & $\begin{array}{c}0.000856 \\
(0.008775)\end{array}$ & $\begin{array}{c}0.02468 \\
(0.002176)\end{array}$ & 1 & $\begin{array}{c}0.005281 \\
(0.000289)\end{array}$ & $\begin{array}{c}0.009626 \\
(0.001758)\end{array}$ & 418.7 & 426.7 & 428.3 & 432.3 & 4.7185 \\
\hline GMW & 0 & $\begin{array}{c}0.5256 \\
(0.06536)\end{array}$ & $\begin{array}{c}0.3819 \\
(0.05393)\end{array}$ & $\begin{array}{c}0.006687 \\
(0.000585)\end{array}$ & $\begin{array}{c}0.06036 \\
(0.01095)\end{array}$ & 355.8 & 363.8 & 365.4 & 369.4 & 0.2396 \\
\hline GAE & $\begin{array}{c}0.006438 \\
(0.003377)\end{array}$ & $\begin{array}{c}0.002438 \\
(0.003435)\end{array}$ & 1 & 0 & $\begin{array}{c}0.677 \\
(0.4661)\end{array}$ & 370.2 & 376.2 & 377.1 & 380.4 & 0.3430 \\
\hline GEV & 0 & 1 & 0 & $\begin{array}{c}0.01024 \\
(0.001629)\end{array}$ & $\begin{array}{c}0.1007 \\
(0.0484)\end{array}$ & 366.3 & 370.3 & 370.7 & 373.1 & 0.2661 \\
\hline GW & 0 & $\begin{array}{c}0.000221 \\
(0.001258)\end{array}$ & $\begin{array}{c}1.453 \\
(0.672)\end{array}$ & 0 & $\begin{array}{l}1.7066 \\
(2.1364)\end{array}$ & 368.3 & 374.3 & 375.2 & 378.5 & 0.3412 \\
\hline BW & $\begin{array}{c}k \\
0.6935 \\
(0.03331)\end{array}$ & $\begin{array}{c}\lambda \\
1.8109 \\
(0.4279)\end{array}$ & $\begin{array}{c}a \\
1.1725 \\
(1.1345)\end{array}$ & $\begin{array}{c}b \\
0.04806 \\
(0.00938)\end{array}$ & & 378.7 & 386.7 & 388.3 & 392.3 & 0.6369 \\
\hline BEW & $\begin{array}{c}k \\
0.9895 \\
(0.03881)\end{array}$ & $\begin{array}{c}\lambda \\
8.0706 \\
(1.1182)\end{array}$ & $\begin{array}{c}\alpha \\
0.7834 \\
(0.2485)\end{array}$ & $\begin{array}{c}a \\
0.9181 \\
(0.5873)\end{array}$ & $\begin{array}{c}b \\
0.0426 \\
(0.00799)\end{array}$ & 371.2 & 381.2 & 383.7 & 388.2 & 0.2904 \\
\hline
\end{tabular}

Fitted PDF

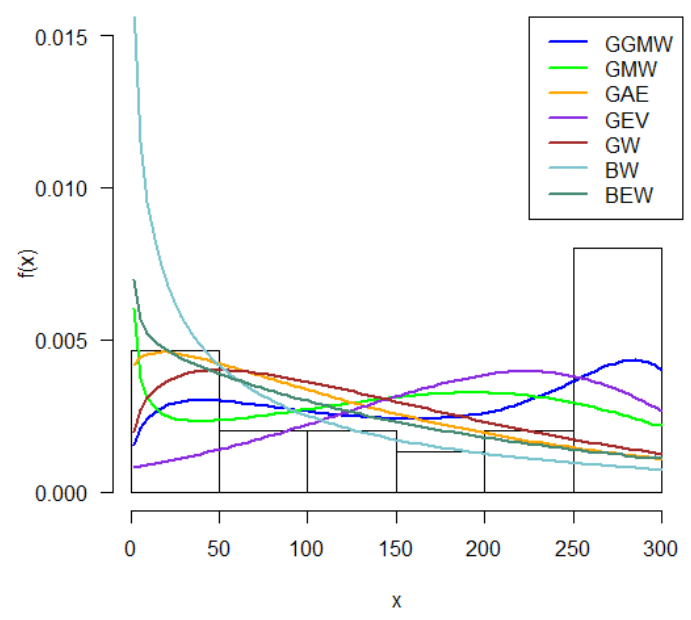

The Graph of Observed vs Expected Probability

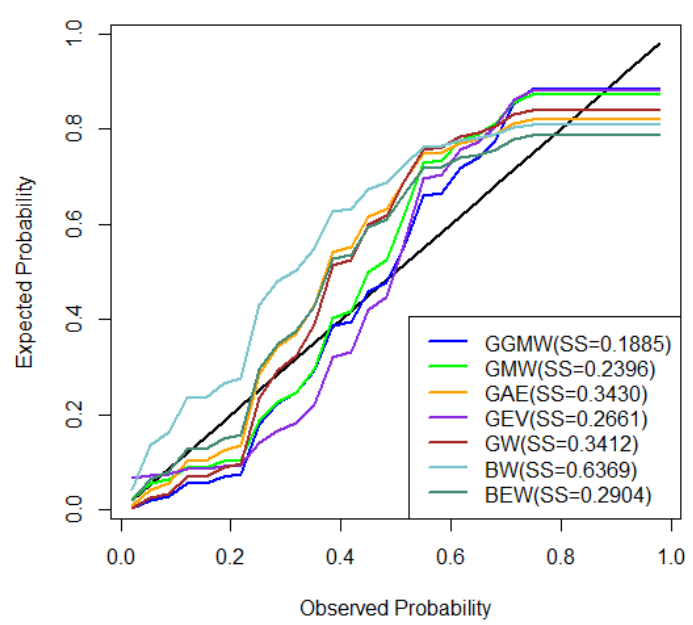

Figure 4: Graphs for meeker data 
Table 6: Estimation of GGMW model for breast cancer data

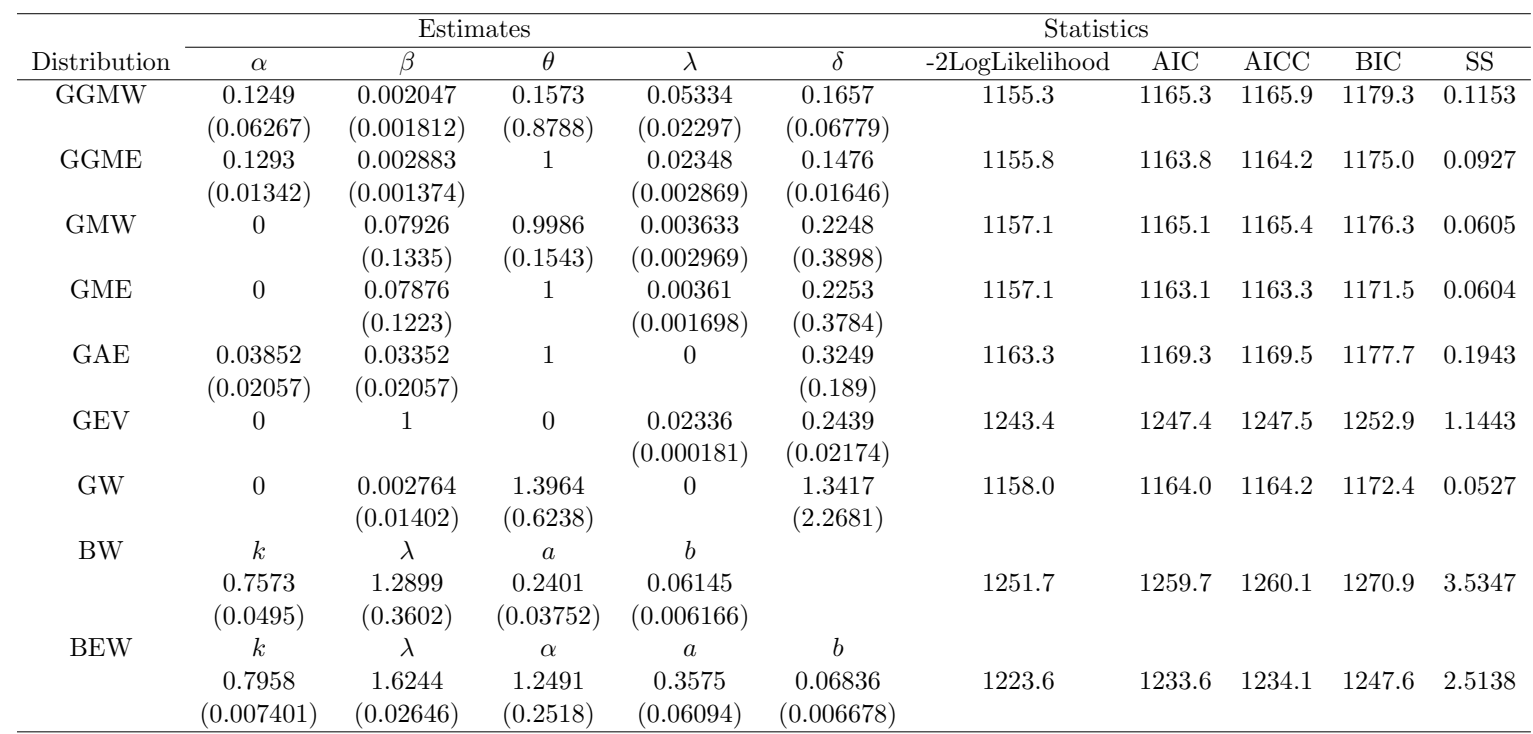

Fitted PDF

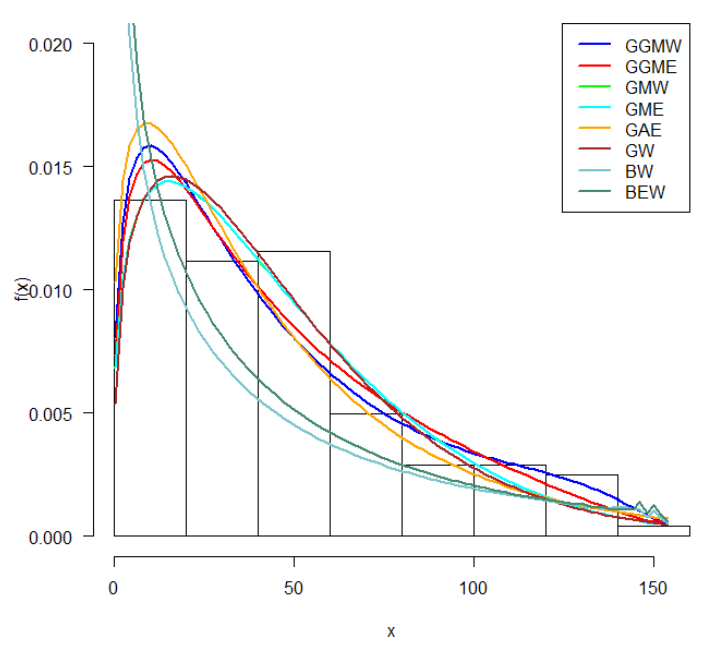

The Graph of Observed vs Expected Probability

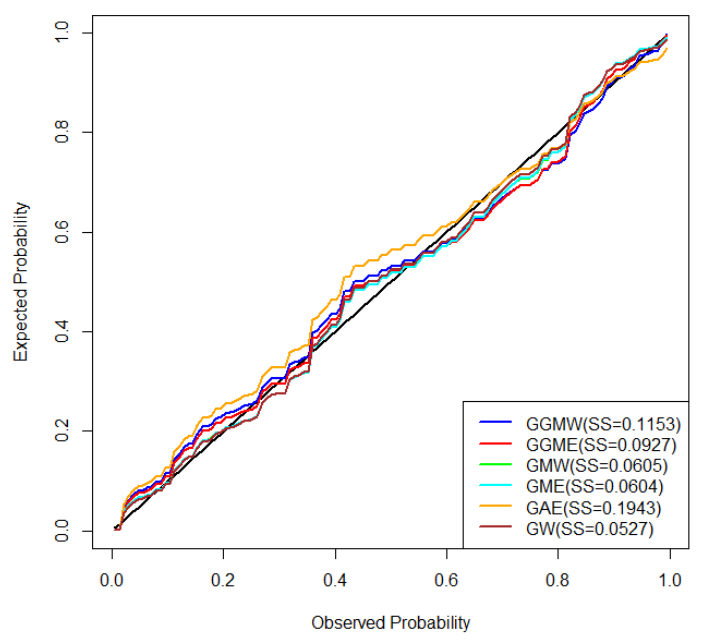

Figure 5: Graphs for breast cancer data 
The estimated covariance matrix for the GGMW distribution (Breast Cancer Data) is given by

$$
\left(\begin{array}{ccccc}
0.003927 & 0.000059 & -0.04903 & 0.001194 & -0.00414 \\
0.000059 & 3.28 E-06 & -0.0009 & 0.000016 & -0.00006 \\
-0.04903 & -0.0009 & 0.7724 & -0.01968 & 0.05052 \\
0.001194 & 0.000016 & -0.01968 & 0.000528 & -0.00122 \\
-0.00414 & -0.00006 & 0.05052 & -0.00122 & 0.004595
\end{array}\right)
$$

Plots of the fitted densities, the histogram of the data are given in Figure 3, Figure 4 and Figure 5. For the probability plot, we plotted $G_{G G M W}\left(x_{(j)} ; \hat{\alpha}, \hat{\beta}, \hat{\theta}, \hat{\lambda}, \hat{\delta}\right)$ against $\frac{j-0.375}{n+0.25}, j=$ $1,2, \cdots, n$, where $x_{(j)}$ are the ordered values of the observed data. We also computed a measure of closeness of each plot to the diagonal line. This measure of closeness is given by the sum of squares

$$
S S=\sum_{j=1}^{n}\left[G_{G G M W}\left(x_{(j)} ; \hat{\alpha}, \hat{\beta}, \hat{\theta}, \hat{\lambda}, \hat{\delta}\right)-\left(\frac{j-0.375}{n+0.25}\right)\right]^{2} .
$$

For waiting times data set, the LR test statistic of the hypothesis $H_{0}$ : GGME against $H_{a}$ : GGMW is $\omega=2.8$. The p-value $=0.094$. Therefore, there is no significant difference between GGMW and GGME distributions at the $5 \%$ level. However, there is a significant difference between GGME and GGMW distributions at the $10 \%$ level. The LR statistic of the hypothesis $H_{0}$ : GEV against $H_{a}$ : GGMW for waiting times data is $\omega=89.4$ The p-value $<0.0001$, we can conclude that there is a significance difference between GGMW and GEV distributions. There is no significant difference between the GGMW and GMW distributions. Also, there is no significant difference between the GW and GMW distributions. The values of the statistics AIC, AICC and BIC shows that the sub-model GW is a good fit for this data. Based on these statistics, the GW distribution could be chosen as the best model among these distributions. The values of the statistics are comparable to those of the non-nested BW distribution and those corresponding to the BEW distribution.

For Meeker data set, the LR test statistics of the hypothesis $H_{0}$ : GGME against $H_{a}$ : GGMW is $\omega=73.5$. The p-value $<0.0001$. Therefore, there is significant difference between GGMW and GGME distributions. The LR statistic of the hypothesis $H_{0}$ : GMW against $H_{a}$ : GGMW is $\omega=10.6$. The p-value $=0.0011$, we can conclude that there is a significance difference between GGMW and GMW distributions. The values of the statistics AIC, BIC, and AICC are smaller for the GGMW distribution. The values of these statistics points to the GGMW distribution as the "better" fit for Meeker data. Also, the values of AIC, BIC and AICC are better for the GMW and GGMW distributions when compared to the non-nested BW and BEW distributions.

For breast cancer data set, there is no significant difference between GGMW, GGME, GMW, GW and GME distributions based on the corresponding LR tests. The sub-models GME and GW seem to be the "best" fits for this data. The values of the statistics AIC, BIC and AICC are smaller for the GME distribution. The values of SS from the probability plots are 0.0604 and 0.0527 for the GME and GW distributions, respectively. The values of these statistics points to and supports the GW as well as the GME distributions as the better fits among the nested distributions. Also, the values of the statistics: AIC, BIC and AICC are far better for the GMW and GGMW distributions when compared to those of the non-nested BW and BEW distributions.

The conclusions based on the LR tests, fitted pdfs, the histograms of the data, and probability plots are in agreement with the statistics AIC, AICC and BIC for the selected models. The GW distribution provides a better fits for the waiting times data, while the GGMW distribution and GME as well as the GW distributions provides better fits for the Meeker and Escobar, and breast cancer data, respectively. 


\section{Concluding remarks}

A new class of generalized modified Weibull distribution called the gamma-generalized modified Weibull (GGMW) distribution is proposed and studied. The GGMW distribution has several sub-models such as the GGMR, GGME, GAE, GLFR, LFR, GMW, GME, MW, MR, ME, Weibull, Raleigh and exponential distributions as special cases. The density of this new class of distributions can be expressed as a linear combination of GMW density functions. The GGMW distribution possesses hazard function with flexible behavior. We also obtain closed form expressions for the moments, distribution of order statistics and Renyi entropy. Maximum likelihood estimation technique was used to estimate the model parameters. Finally, the GGMW distribution and its sub-models was fitted to real data sets to illustrate the applicability and usefulness of this class of distributions.

\section{Acknowledgements}

The authors would like to thank the editor and the referee for carefully reading the paper and for their valuable comments, which greatly improved the presentation in this paper.

\section{References}

Almalki SJ, Yuan J (2013). "A New Modified Weibull Distribution." Reliability Engineering and System Safety, 111, 164-170.

Bain L (1974). "Analysis for the Linear Failure Rate Life Testing Distribution." Technometrics, 16(4), 551-559.

Barlow R, Campo R (1975). Total Time on Test Processes and Applications to Failure Data Analysis. Society for Industrial and Applied Mathematics.

Bebbington M, Lai C, Zitikis R (2007). "A Flexible Weibull Extension.” Reliability Engineering and System Safety, 92(6), 719-726.

Carrasco M, Ortega EM, Cordeiro G (2008). "A Generalized Weibull Distribution for Lifetime Modeling." Computational Statistics and Data Analysis, 53(2), 450-462.

Choudhury A (2005). "A Simple Derivation of Moments of the Exponentiated Weibull Distribution." Metrika, 62(1), 17-22.

Cordeiro G, Gomes A, da Silva C, Ortega M (2013). "The Beta Exponentiated Weibull Distribution." Journal of Statistical Computation and Simulations, 38(1), 114-138.

Cordeiro G, Ortega E, Nadarajah S (2010). "The Kumaraswamy Weibull Distribution with Applications to Failure Data." Journal of Franklin Institute, 347(8), 1399-1429.

Famoye F, Lee C, Olumolade O (2005). "The Beta-Weibull Distribution." Journal of Statistical Theory and Applications, pp. 121-138.

Ferguson T (1996). A Course in Large Sample Theory. Chapman and Hall.

Fisher R (1934). "The Effects of Methods of Ascertainment Upon the Estimation of Frequencies." Annals of Human Genetics, 6(1), 439 - 444.

Ghitany M, Atieh B, Nadarajah S (2008). "Lindley Distribution and Its Applications." Mathematics and Computers in Simulations, 78(4), 493-506.

Gradshteyn I, Ryzhik I (2000). Tables of Integrals, Series and Products. Academic Press.

Gupta R, Keating J (1985). "Relation for Reliability Measures under Length Biased Sampling." Scandinavian Journal of Statistics, 13, 49-56. 
Gupta R, Kundu D (1999). "Generalized Exponential Distributions." Australian and New Zealand Journal of Statistics, 43, 117-130.

Gupta R, Kundu D (2001). "Exponentiated Exponential Distribution: An Alternative to Gamma and Weibull Distributions." Biometrical Journal, 43, 117-130.

Haupt E, Schabe H (1992). "A New Model for A Lifetime Distribution with Bathtub Shaped Failure Rate." Microelectronics and Reliability, 32, 633-639.

Hjorth U (1980). "A Reliability Distribution with Increasing, Decreasing, Constant and Bathtub Failure Rates." Technometrics, 22, 99-107.

Kundu D, Rakab M (2005). "Generalized Rayleigh Distribution: Different Methods of Estimation." Computational Statistics and Data Analysis, 49, 187-200.

Lai C, Moore T, Xie M (1998). "The Beta Integrated Model." Proceedings International Workshop on Reliability Modeling and Analysis-From Theory to Practice, pp. 153-159.

Lai C, Xie M, Murthy D (2003). "A Modified Weibull Distribution." IEEE Transactions on Reliability, 52, 33-37.

Lee C, Famoye F, Olumolade O (2007). "Beta Weibull Distribution, Properties and Applications to Censored Data." Journal of Mod. Appl. Statist. Meth, 6, 173-186.

Lee E (1992). Statistical Methods for Survival Data Analysis. John Wiley.

Marshall AW, Olkin I (1997). "A New Method for Adding a Parameter to a Family of Distributions with Applications to the Exponential and Weibull Families." Biometrika, 84(3), 641-652.

Meeker W, Escobar L (1998). Statistical Methods for Reliability Data. John Wiley.

Mudholkar G, Srivastava D, Friemer M (1995). "The Exponentiated Weibull Family: A Reanalysis of the Bus-motor-failure Data." Technometrics, 37, 436-445.

Mudholkar G, Srivastava D, Kollia G (1996). "A Generalization of the Weibull Distribution with Application to the Analysis of Survival Data." Journal of the American Statistical Association, 91, 1575-1583.

Nadarajah S (2005). "On the Moments of the Modified Weibull Distribution." Reliability Engineering and System Safety, 90, 114-117.

Nadarajah S, Cordeiro GM, Ortega EMM (2011). "General Results for the beta-Modified Weibull Distribution." Journal of Statistical Computation and Simulation, 81(10), 12111232 .

Nadarajah S, Kotz S (2005). "On Some Recent Modifications of Weibull Distribution." IEEE Transactions Reliability, 54, 561-562.

Nanda K, Jain K (1999). "Some Weighted Distribution Results on Univariate and Bivariate Cases." Journal of Statistical Planning and Inference, 77(2), 169 - 180.

Nassar M, Eissa F (2003). "On the Exponentiated Weibull Distribution." Communications in Statistics - Theory and Methods, 32(7), 1317-1336.

Oluyede B (1999). "On Inequalities and Selection of Experiments for Length-Biased Distributions." Probability in the Engineering and Informational Sciences, 13(2), 129-145.

Patil G, Rao C (1978). "Weighted Distributions and Size-Biased Sampling with Applications to Wildlife and Human Families." Biometrics, 34(6), 179-189. 
Phani KK (1987). "A New Modified Weibull Distribution Function." Communications of the American Ceramic Society, 70(8), 182-184.

Pinho L, Cordeiro G, Nobre J (2012). "The Gamma-Exponentiated Weibull Distribution." Journal of Statistical Theory and Applications, 11(4), 379-395.

Rajarshi S, Rajarshi M (1988). "Bathtub Distributions: A Review." Communications in Statistics-Theory and Methods, 17, 2521-2597.

Rao C (1965). "On Discrete Distributions Arising out of Methods of Ascertainment." The Indian Journal of Statistics, 27(2), 320 - 332.

Ristić M, Balakrishnan N (2011). "The Gamma-Exponentiated Exponential Distribution." J. Statist. Comp. and Simulation, 82(8), 1191-1206.

Santos Silva JMC, Tenreyro S (2010). "On the Existence of Maximum Likelihood Estimates in Poisson Regression." Economics Letters, 107, 310-312.

Sarhan AM, Zaindin M (2009). "Modified Weibull Distribution." Applied Sciences, 11, 123136.

Seregin A (2010). "Uniqueness of the Maximum Likelihood Estimator for K-monotone Densities." Proceedings of the American Mathematical Society, 138(12), 4511-4515.

Shaked M, Shanthikumar J (1994). Stochastic Orders and Their Applications. Academic Press.

Silva G, Ortega E, Cordeiro G (2010). "The Beta Modified Weibull Distribution." Lifetime Data Analysis, 16, 409-430.

Singha N, Jain K, Kumar SS (2012). "The Beta Generalized Weibull Distribution: Properties and Applications." Reliability Engineering and System Safety, 102, 5-15.

The R Development Core Team (2011). "A Language and Environment for Statistical Computing." $R$ Foundation for Statistical Computing.

Weibull WA (1951). "Statistical Distribution Function of Wide Applicability." Journal of Applied Mechanics, 18, 293-296.

Xia J, Mi J, Zhou YY (2009). "On the Existence and Uniqueness of the Maximum Likelihood Estimators of Normal and Log-normal Population Parameters with Grouped Data." Journal of Probability and Statistics.

Xie M, Lai C (1995). "Reliability Analysis Using an Additive Weibull Model with Bathtubshaped Failure Rate Function." Reliability Engineering and System Safety, 52, 87-93.

Xie M, Tang Y, Goh T (2002). "A Modified Weibull Extension with Bathtub Failure Rate Function." Reliability Engineering and System Safety, 76, 279-285.

Zhang T, Xie M (2011). "On the Upper Truncated Weibull Distribution and Its Reliability Implications." Reliability Engineering and System Safety, 96(1), 194-200.

Zhou C (2009). "Existence and Consistency of the Maximum Likelihood Estimator for the Extreme Index." J. Multivariate Analysis, 100, 794-815.

Zografos K, Balakrishnan N (2009). "On Families of Beta- and Generalized Gamma-Generated Distribution and Associated Inference." Stat. Method, 6, 344-362. 


\section{APPENDIX}

Let $A\left(x_{i} ; \alpha, \beta, \theta, \lambda\right)=\left(1-e^{-\alpha x_{i}-\beta x_{i}^{\theta} e^{\lambda x_{i}}}\right) \log \left(1-e^{-\alpha x_{i}-\beta x_{i}^{\theta} e^{\lambda x_{i}}}\right), B\left(x_{i} ; \alpha, \beta, \theta, \lambda\right)=e^{-\alpha x_{i}-\beta x_{i}^{\theta} e^{\lambda x_{i}}}+$ $\log \left(1-e^{-\alpha x_{i}-\beta x_{i}^{\theta} e^{\lambda x_{i}}}\right)$, and $C\left(x_{i} ; \alpha, \beta, \theta, \lambda\right)=\left(1-e^{-\alpha x_{i}-\beta x_{i}^{\theta} e^{\lambda x_{i}}}-\beta x_{i}^{\theta} e^{\lambda x_{i}}\right) \log \left(1-e^{-\alpha x_{i}-\beta x_{i}^{\theta} e^{\lambda x_{i}}}\right)-$ $\beta x_{i}^{\theta} e^{-(\alpha-\lambda) x_{i}-\beta x_{i}^{\theta} e^{\lambda x_{i}}}$. Elements of the observed information matrix of the GGMW distribution are given by

$$
\begin{aligned}
& \frac{\partial^{2} \ell}{\partial \alpha^{2}}=\sum_{i=1}^{n} \frac{(1-\delta) x_{i}^{2} e^{-\alpha x_{i}-\beta x_{i}^{\theta} e^{\lambda x_{i}}} B\left(x_{i} ; \alpha, \beta, \theta, \lambda\right)}{A^{2}\left(x_{i} ; \alpha, \beta, \theta, \lambda\right)} \\
& -\sum_{i=1}^{n} \frac{1}{\left[\alpha+\beta x_{i}^{\theta-1} e^{\lambda x_{i}}\left(\theta+\lambda x_{i}\right)\right]^{2}} . \\
& \frac{\partial^{2} \ell}{\partial \alpha \partial \beta}=\sum_{i=1}^{n} \frac{(1-\delta) x_{i}^{\theta+1} e^{-(\alpha-\lambda) x_{i}-\beta x_{i}^{\theta} e^{\lambda x_{i}} B\left(x_{i} ; \alpha, \beta, \theta, \lambda\right)}}{A^{2}\left(x_{i} ; \alpha, \beta, \theta, \lambda\right)} \\
& -\sum_{i=1}^{n} \frac{x_{i}^{\theta-1} e^{\lambda x_{i}}\left(\theta+\lambda x_{i}\right)}{\left[\alpha+\beta x_{i}^{\theta-1} e^{\lambda x_{i}}\left(\theta+\lambda x_{i}\right)\right]^{2}} . \\
& \frac{\partial^{2} \ell}{\partial \alpha \partial \theta}=\sum_{i=1}^{n} \frac{(1-\delta) \beta x_{i}^{\theta+1} e^{-(\alpha-\lambda) x_{i}-\beta x_{i}^{\theta} e^{\lambda x_{i}}} \log \left(x_{i}\right) B\left(x_{i} ; \alpha, \beta, \theta, \lambda\right)}{A^{2}\left(x_{i} ; \alpha, \beta, \theta, \lambda\right)} \\
& -\sum_{i=1}^{n} \frac{\beta x_{i}^{\theta-1} e^{\lambda x_{i}}\left[\left(\theta+\lambda x_{i}\right) \log \left(x_{i}\right)+1\right]}{\left[\alpha+\beta x_{i}^{\theta-1} e^{\lambda x_{i}}\left(\theta+\lambda x_{i}\right)\right]^{2}} . \\
& \frac{\partial^{2} \ell}{\partial \alpha \partial \lambda}=\sum_{i=1}^{n} \frac{(1-\delta) \beta x_{i}^{\theta+2} e^{-(\alpha-\lambda) x_{i}-\beta x_{i}^{\theta} e^{\lambda x_{i}} B\left(x_{i} ; \alpha, \beta, \theta, \lambda\right)}}{A^{2}\left(x_{i} ; \alpha, \beta, \theta, \lambda\right)} \\
& -\sum_{i=1}^{n} \frac{\beta x_{i}^{\theta} e^{\lambda x_{i}}\left(\theta+\lambda x_{i}+1\right)}{\left[\alpha+\beta x_{i}^{\theta-1} e^{\lambda x_{i}}\left(\theta+\lambda x_{i}\right)\right]^{2}} . \\
& \frac{\partial^{2} \ell}{\partial \alpha \partial \delta}=\sum_{i=1}^{n} \frac{e^{-\alpha x_{i}-\beta x_{i}^{\theta} e^{\lambda x_{i}}} x_{i}}{A\left(x_{i} ; \alpha, \beta, \theta, \lambda\right)} . \\
& \frac{\partial^{2} \ell}{\partial \beta^{2}}=\sum_{i=1}^{n} \frac{(1-\delta) x_{i}^{2 \theta} e^{-(\alpha-2 \lambda) x_{i}-\beta x_{i}^{\theta} e^{\lambda x_{i}} B\left(x_{i} ; \alpha, \beta, \theta, \lambda\right)}}{A^{2}\left(x_{i} ; \alpha, \beta, \theta, \lambda\right)} \\
& -\sum_{i=1}^{n} \frac{x_{i}^{2 \theta-2} e^{2 \lambda x_{i}}\left(\theta+\lambda x_{i}\right)^{2}}{\left[\alpha+\beta x_{i}^{\theta-1} e^{\lambda x_{i}}\left(\theta+\lambda x_{i}\right)\right]^{2}} . \\
& \frac{\partial^{2} \ell}{\partial \beta \partial \theta}=\sum_{i=1}^{n} \frac{(\delta-1) x_{i}^{\theta} e^{-(\alpha-\lambda) x_{i}-\beta x_{i}^{\theta} e^{\lambda x_{i}}} \log \left(x_{i}\right) C\left(x_{i} ; \alpha, \beta, \theta, \lambda\right)}{A^{2}\left(x_{i} ; \alpha, \beta, \theta, \lambda\right)} \\
& +\sum_{i=1}^{n} \frac{x_{i}^{\theta-1} e^{\lambda x_{i}}\left[\left(\theta+\lambda x_{i}\right) \log \left(x_{i}\right)+1\right] \alpha}{\left[\alpha+\beta x_{i}^{\theta-1} e^{\lambda x_{i}}\left(\theta+\lambda x_{i}\right)\right]^{2}}-\sum_{i=1}^{n} x_{i}^{\theta} e^{\lambda x_{i}} \log \left(x_{i}\right) .
\end{aligned}
$$




$$
\begin{aligned}
& \frac{\partial^{2} \ell}{\partial \beta \partial \lambda}=\sum_{i=1}^{n} \frac{(\delta-1) x_{i}^{\theta+1} e^{-(\alpha-\lambda) x_{i}-\beta x_{i}^{\theta}{ }^{\lambda x_{i}} C\left(x_{i} ; \alpha, \beta, \theta, \lambda\right)}}{A^{2}\left(x_{i} ; \alpha, \beta, \theta, \lambda\right)} \\
& +\sum_{i=1}^{n} \frac{x_{i}^{\theta} e^{\lambda x_{i}}\left(\theta+\lambda x_{i}+1\right) \alpha}{\left[\alpha+\beta x_{i}^{\theta-1} e^{\lambda x_{i}}\left(\theta+\lambda x_{i}\right)\right]^{2}}-\sum_{i=1}^{n} x_{i}^{\theta+1} e^{\lambda x_{i}} . \\
& \frac{\partial^{2} \ell}{\partial \beta \partial \delta}=\sum_{i=1}^{n} \frac{e^{-(\alpha-\lambda) x_{i}-\beta x_{i}^{\theta} e^{\lambda x_{i}}} x_{i}^{\theta}}{A\left(x_{i} ; \alpha, \beta, \theta, \lambda\right)} \\
& \frac{\partial^{2} \ell}{\partial \theta^{2}}=\sum_{i=1}^{n} \frac{(\delta-1) \beta x_{i}^{\theta} e^{-(\alpha-\lambda) x_{i}-\beta x_{i}^{\theta} e^{\lambda x_{i}}}\left(\log \left(x_{i}\right)\right)^{2} C\left(x_{i} ; \alpha, \beta, \theta, \lambda\right)}{A^{2}\left(x_{i} ; \alpha, \beta, \theta, \lambda\right)} \\
& +\sum_{i=1}^{n} \frac{\beta x_{i}^{\theta-1} e^{\lambda x_{i}}\left\{\left[\left(\theta+\lambda x_{i}\right)\left(\log \left(x_{i}\right)\right)^{2}+2 \log \left(x_{i}\right)\right] \alpha-\beta x_{i}^{\theta-1} e^{\lambda x_{i}}\right\}}{\left[\alpha+\beta x_{i}^{\theta-1} e^{\lambda x_{i}}\left(\theta+\lambda x_{i}\right)\right]^{2}} \\
& -\beta \sum_{i=1}^{n} x_{i}^{\theta} e^{\lambda x_{i}}\left(\log \left(x_{i}\right)\right)^{2} \\
& \frac{\partial^{2} \ell}{\partial \theta \partial \lambda}=\sum_{i=1}^{n} \frac{(\delta-1) \beta x_{i}^{\theta+1} e^{-(\alpha-\lambda) x_{i}-\beta x_{i}^{\theta} e^{\lambda x_{i}}} \log \left(x_{i}\right) C\left(x_{i} ; \alpha, \beta, \theta, \lambda\right)}{A^{2}\left(x_{i} ; \alpha, \beta, \theta, \lambda\right)} \\
& +\sum_{i=1}^{n} \frac{\beta x_{i}^{\theta} e^{\lambda x_{i}}\left\{\left[\left(\theta+\lambda x_{i}+1\right) \log \left(x_{i}\right)+1\right] \alpha-\beta x_{i}^{\theta-1} e^{\lambda x_{i}}\right\}}{\left[\alpha+\beta x_{i}^{\theta-1} e^{\lambda x_{i}}\left(\theta+\lambda x_{i}\right)\right]^{2}} \\
& -\beta \sum_{i=1}^{n} x_{i}^{\theta+1} e^{\lambda x_{i}} \log \left(x_{i}\right) \\
& \frac{\partial^{2} \ell}{\partial \theta \partial \delta}=\sum_{i=1}^{n} \frac{\beta e^{-(\alpha-\lambda) x_{i}-\beta x_{i}^{\theta} e^{\lambda x_{i}}} x_{i}^{\theta} \log \left(x_{i}\right)}{A\left(x_{i} ; \alpha, \beta, \theta, \lambda\right)} \\
& \frac{\partial^{2} \ell}{\partial \lambda^{2}}=\sum_{i=1}^{n} \frac{(\delta-1) \beta x_{i}^{\theta+2} e^{-(\alpha-\lambda) x_{i}-\beta x_{i}^{\theta} e^{\lambda x_{i}} C\left(x_{i} ; \alpha, \beta, \theta, \lambda\right)}}{A^{2}\left(x_{i} ; \alpha, \beta, \theta, \lambda\right)} \\
& +\sum_{i=1}^{n} \frac{\beta x_{i}^{\theta+1} e^{\lambda x_{i}}\left[\left(\theta+\lambda x_{i}+2\right) \alpha-\beta x_{i}^{\theta-1} e^{\lambda x_{i}}\right]}{\left[\alpha+\beta x_{i}^{\theta-1} e^{\lambda x_{i}}\left(\theta+\lambda x_{i}\right)\right]^{2}}-\beta \sum_{i=1}^{n} x_{i}^{\theta+2} e^{\lambda x_{i}} . \\
& \frac{\partial^{2} \ell}{\partial \lambda \partial \delta}=\sum_{i=1}^{n} \frac{\beta e^{-(\alpha-\lambda) x_{i}-\beta x_{i}^{\theta} e^{\lambda x_{i}} x_{i}^{\theta+1}}}{A\left(x_{i} ; \alpha, \beta, \theta, \lambda\right)} \\
& \frac{\partial^{2} \ell}{\partial \delta^{2}}=-n \Psi^{\prime}(\delta), \quad \text { where } \quad \Psi(\delta)=\frac{d \log (\Gamma(\delta))}{d \delta}=\frac{\Gamma^{\prime}(\delta)}{\Gamma(\delta)}
\end{aligned}
$$




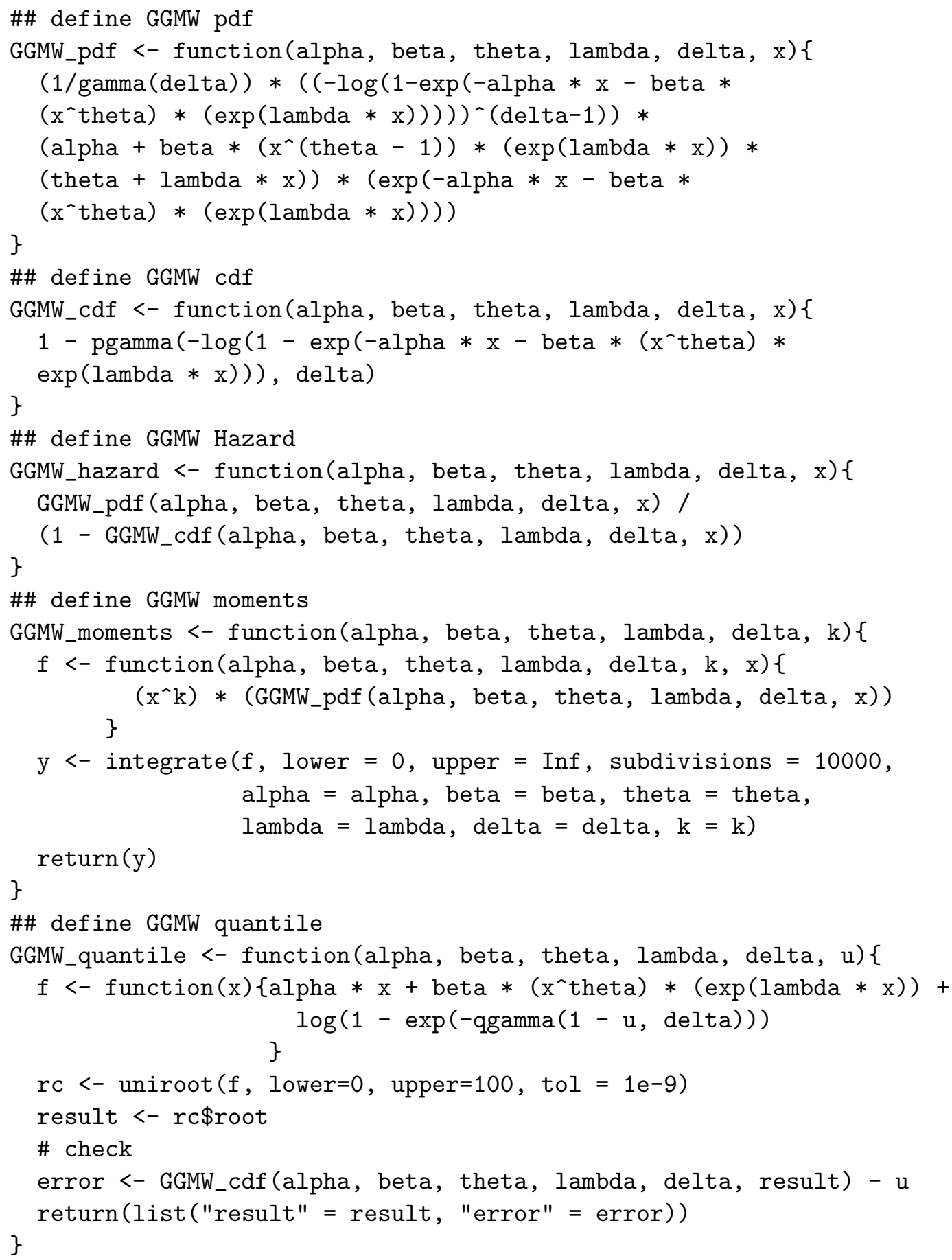

\section{Affiliation:}

Broderick O. Oluyede

Department of Mathematical Sciences

Georgia Southern University

Statesboro, GA 30460

E-mail: boluyede@georgiasouthern.edu

\section{Austrian Journal of Statistics}

published by the Austrian Society of Statistics

Volume 44

October 2015 http://www.ajs.or.at/

http://www.osg.or.at/

Submitted: 2014-05-12

Accepted: 2015-01-27 